Published in final edited form as:

Organometallics. 2007 ; 26(2): 281-287. doi:10.1021/om0606643.

\title{
Reactions of Indene and Indoles with Platinum Methyl Cations: Indene C-H Activation, Indole $\pi$ vs. Nitrogen Lone-Pair Coordination
}

\author{
Travis J. Williams, Jay A. Labinger, and John E. Bercaw \\ Arnold and Mabel Beckman Laboratories of Chemical Synthesis, California Institute of \\ Technology, Pasadena, CA 91125 (U. S. A.)
}

\begin{abstract}
Reactions of indene and various substituted indoles with [(diimine) $\left.\mathrm{Pt}^{\mathrm{II}}(\mathrm{Me})(\mathrm{TFE})\right]^{+}$cations have been studied (diimine $=\operatorname{ArN}=\mathrm{C}(\mathrm{Me})-\mathrm{C}(\mathrm{Me})=\mathrm{NAr}$; $\mathrm{TFE}=2,2,2$-trifluoroethanol). Indene displaces the TFE ligand from platinum to form a stable $\pi$ coordination complex that, upon heating, undergoes C-H activation with first order kinetics, $\Delta H^{t}=29 \mathrm{kcal} / \mathrm{mol}, \Delta S^{\ddagger}=10 \mathrm{eu}$, and a kinetic isotope effect of 1.1 at $60{ }^{\circ} \mathrm{C}$. Indoles also initially form coordination complexes through the $\mathrm{C} 2=\mathrm{C} 3$ olefin, but these undergo rearrangement to the corresponding $\mathrm{N}$-bound complexes. The relative rates of initial coordination and rearrangement are affected by excess acid or methyl substitution on indole.
\end{abstract}

\section{Introduction}

Selective C-H bond activation is a potentially valuable approach to synthetic problems in areas ranging from fuels and bulk chemicals to fine chemicals and pharmaceutical synthesis. 1 Studies of C-H activation in our laboratory have focused on models of the Shilov system,2 particularly $\left[(\text { diimine }) \mathrm{Pt}^{\mathrm{II}}(\mathrm{Me})(\text { solv })\right]^{+}(\mathbf{2}$, diimine $=\mathrm{ArN}=\mathrm{C}(\mathrm{Me})-\mathrm{C}(\mathrm{Me})=\mathrm{NAr}$; solv $=$ 2,2,2-trifluoroethanol (TFE), $\mathrm{H}_{2} \mathrm{O}$ ). 3 These cations are capable of activating a variety of carbon-hydrogen bonds. 4 Cations 2 can be generated by protonolysis of (diimine) $\mathrm{Pt}^{\mathrm{II}} \mathrm{Me}_{2}$ species 1 in TFE with aqueous $\mathrm{HBF}_{4} 3,4 \mathrm{ab}$ or $\mathrm{BX}_{3}\left(\mathrm{X}=\mathrm{C}_{6} \mathrm{~F}_{5}, 4 \mathrm{~cd} \mathrm{~F} 5\right)$, the latter producing $\mathrm{H}^{+}$by boron coordination to TFE. $4 \mathrm{c}$ In deuterated solvent $\mathbf{2}$ is formed as a mixture of two isotopologs. Reaction of $\mathbf{2}$ with a $\mathrm{C}-\mathrm{H}$ group then results in liberation of methane as a mixture of $\mathrm{CH}_{4}$ and $\mathrm{CH}_{3} \mathrm{D}$ along with the formation of a new alkyl or aryl platinum(II) complex [(diimine) $\mathrm{Pt}^{\mathrm{II}}(\mathrm{R})($ solv $\left.)\right]^{+}$(Scheme 1$) .6$

The presence of donor heteroatoms $(\mathrm{N}, \mathrm{O}, \mathrm{S}$, etc.) in the substrate may inhibit $\mathrm{C}-\mathrm{H}$ activation because these heteroatoms tend to bind tightly to electrophilic metal centers and block $\mathrm{C}-\mathrm{H}$ bond coordination. In the cases of methanol and dimethyl ether,4e stable complexes of the organic substrate with platinum are observed, but kinetics studies indicate that substrateplatinum binding is rapid and reversible, and that re-binding (or rearrangement) of the substrate to a $\mathrm{C}-\mathrm{H}$ bond-coordinated intermediate is rate-determining. Heteroatom binding can be used to advantage, however, in coordinatively directed cyclometallation reactions, 7 the basis of many synthetic methods. Several recent examples of these involving nitrogencontaining heterocycles have appeared. 8 Specifically regarding indole, a functionality found 
frequently in natural products and pharmaceutical agents, several groups have identified conditions for cyclization of indoles with pendant olefins, 9 and both Sames10 and Sanford 11 have reported selective arylation of simple indoles via palladium-catalyzed C-H activation.

Prior literature reports of stable group 10 metal-indole complexes are few in number. Platinum-coordinated tryptophan and tryptamine derivatives have been studied, 12 but only a single platinum complex, cis-Pt(indole) ${ }_{2} \mathrm{Cl}_{2}$, having a neutral, simple L-type donor indole ligand13 has been reported. Unfortunately, it was characterized only by elemental analysis and infrared, and neither offers insight into the binding of the indole ligand.14 Moreover, although (1-indolyl)metal amides (functioning as X-type ligands) are common,15 and indole-metal arene complexes have been described for several transition metals, 16 we are aware of no examples of transition metal complexes having indole $\mathrm{C} 2=\mathrm{C} 3$ olefin ligation.

The present work examines the reactions of indoles with cations $\mathbf{2}$. Towards this end, we describe the reaction of these cations with indene, indole's hydrocarbon analog, as well as with various methyl-substituted indoles. We find that indene forms a stable olefin coordination complex that undergoes $\mathrm{C}-\mathrm{H}$ activation upon heating. Analogously, indoles initially form olefin coordination complexes, but rather than undergoing $\mathrm{C}-\mathrm{H}$ activation, these complexes rearrange to stable $\mathrm{N}$-bound $3 \mathrm{H}$-indole species. We expect that $\mathrm{C}-\mathrm{H}$ activation of nitrogen-containing unsaturated heterocycles is more likely to proceed from a metal-heterocycle $\pi$ complex than an $N$-complex, so strategies to extend the lifetime of platinum(II)-indole $\pi$ complexes have been pursued. We find that the rate of rearrangement of indole $\pi$ complexes to $\mathrm{N}$-complexes can be diminished by introducing steric bulk on the indole or by increasing $\left[\mathrm{H}^{+}\right]$.

\section{Results and Discussion}

\section{Reactions of Indene with Cations 2}

Treatment of platinum cations 2 with one equivalent of indene results in the formation of stable olefin complexes, $\mathbf{3}$ (Scheme 2). In each case, complexation is efficient and rapid. Exchange of coordinated indene with 1 equivalent $(15 \mathrm{mM})$ of added indene- $d_{3}$ under the same conditions is slower, with $k_{\mathrm{obs}} \sim 5 \times 10^{-3} \mathrm{~s}^{-1}, \mathrm{t}_{1 / 2} \sim 1 \mathrm{~min}$ (from McKay analysis). 17 The resulting products 3 can be characterized by NMR and electrospray MS methods. The ${ }^{1} \mathrm{H}$ NMR spectrum for 3a is sharp at ambient temperature, and ${ }^{195} \mathrm{Pt}-\mathrm{H}$ coupling constants of 74 and $79 \mathrm{~Hz}$ are observed for the $\mathrm{C} 2$ and $\mathrm{C} 3$ protons of indene. The $\mathrm{C} 1$ methylene protons appear as a pair of doublets $\left(2 J_{\mathrm{H}-\mathrm{H}}=23 \mathrm{~Hz}\right)$ with weak ${ }^{195} \mathrm{Pt}$ side bands $\left(J_{\mathrm{Pt}-\mathrm{H}}=41 \mathrm{~Hz}\right)$. By contrast, ambient temperature ${ }^{1} \mathrm{H}$ NMR spectra of $\mathbf{3 b}, \mathbf{3} \mathbf{c}$, and $\mathbf{3 d}$ give broad signals for the $\mathrm{C} 1, \mathrm{C} 2$, and $\mathrm{C} 3$ indenyl $\mathrm{C}-\mathrm{H}$ protons near $3.2,6.5$, and $5.6 \mathrm{ppm}$, respectively. In all cases, $\mathrm{Pt}-\mathrm{CH}_{3}$ signals were shifted significantly upfield from the parent cation 2: whereas 2 typically displays $\delta\left(\mathrm{Pt}-\mathrm{CH}_{3}\right)$ in the range $0.6-1.3 \mathrm{ppm}$, indene complexes 3 have $\delta\left(\mathrm{Pt}-\mathrm{CH}_{3}\right)$ between -0.8 and $-0.3 \mathrm{ppm}$. This shift is similar to that for a previously-reported $\eta 2$-benzene adduct, which has $\delta\left(\mathrm{Pt}-\mathrm{CH}_{3}\right)=-1.3 \mathrm{ppm}$ at $-33{ }^{\circ} \mathrm{C} .4 \mathrm{a}$ Upon gentle heating, complexes $\mathbf{3}$ react to form $\eta 3$-indenyl complex $\mathbf{4}$ and methane (Scheme 2). In the case of $\mathbf{3 a}$, the reaction is clean (> 95\% yield) as determined by ${ }^{1} \mathrm{H}$ NMR. By 
contrast, heating complexes $\mathbf{3 b}$ and $\mathbf{3 c}$ results in formation of a complex mixture of products, including 4 .

\section{Mechanism of Indene Activation}

First-order rate constants were measured for the conversion of indene complex $\mathbf{3 a}$ to indenyl complex 4a (Table 1). Importantly, two trials at $60{ }^{\circ} \mathrm{C}$ with 1 and 5 equivalents of indene gave the same rate constants $\left(1.24(9) \times 10^{-4} \mathrm{~s}^{-1}\right.$ and $\left.1.37(9) \times 10^{-4} \mathrm{~s}^{-1}\right)$ within error, indicating a zero order rate dependence on [indene]. Activation parameters for the conversion of 3a to 4a, measured by Eyring analysis for kinetic runs conducted at temperatures ranging from 50 to $90{ }^{\circ} \mathrm{C}$ (Figure 1), are $\Delta H^{t}=28.7 \pm 0.7 \mathrm{kcal} \cdot \mathrm{mol}^{-1}$ and $\Delta S^{t}$ $=9.7 \pm 2.2$ e.u. 18 A kinetic isotope effect was determined for the conversion indene- $d_{3}$ to deuterated indenyl complex 4a- $d_{2}$ (Scheme 3). 1,1,3-Trideuteroindene was prepared according to a previously reported procedure 19 and was complexed to $\mathbf{2 a}$ as above. Conversion of $\mathbf{3 a}-d_{3}$ to $\mathbf{4 a}-d_{2}$ proceeded smoothly to afford a selectively deuterated product. Comparisons of measured rate constants for parallel runs gave a kinetic isotope effect of 1.1.

The small, positive value for $\Delta S^{t}$ and the small KIE are suggestive of an intramolecular 3ato-4a process not involving rate-determining $\mathrm{C}-\mathrm{H}$ oxidative addition. A mechanistic scenario that is in accord with these features involves rate-determining rearrangement of $\pi$ complex 3a to $\mathrm{C}-\mathrm{H} \sigma$ complex $\mathbf{5}$, followed by more rapid $\mathrm{C}-\mathrm{H}$ oxidative cleavage and reductive elimination of methane (Scheme 4). Analogous reaction of $\mathbf{2 e}\left(\operatorname{solv}=\mathrm{CF}_{3} \mathrm{CD}_{2} \mathrm{OD}\right)$ with $p$ xylene was found to afford a mixture of products resulting from competitive aryl $\left(k_{\mathrm{H}} / k_{\mathrm{D}} \approx\right.$ $4)$ and benzylic $\left(k_{\mathrm{H}} / k_{\mathrm{D}} \approx 1\right) \mathrm{C}-\mathrm{H}$ activation processes, the latter affording an $\eta 3$-benzylic product. $4 \mathrm{c}$ Rearrangement of $\mathbf{3 a}$ to $\mathbf{4 a}$ thus resembles the benzylic activation process, and suggests that benzylic $\mathrm{C}-\mathrm{H}$ bond activation by $\mathbf{2 e}$ also proceeds via an initially formed arene $\pi$ complex that undergoes rate-determining rearrangement to a $\mathrm{C}-\mathrm{H} \sigma$ complex, followed by $\mathrm{C}-\mathrm{H}$ oxidative cleavage.

\section{Reactions of Cations 2 with Indoles}

Indole reacts rapidly with platinum cations $\mathbf{2}$, ultimately to give $N$-complexation products 8 (Scheme 5). Analogously to reaction with indene, the initially formed adduct is a $\mathrm{C} 2=\mathrm{C} 3 \pi$ complex 6; however, $\mathrm{N}$-ligation, likely via transient 7, inhibits $\mathrm{C}-\mathrm{H}$ activation, and tautomer $\mathbf{8}$ is the stable adduct that is generated.20 Reaction of $\mathbf{2 a}$ with indole at $40{ }^{\circ} \mathrm{C}$ begins with a buildup of $6 \mathbf{a}-d_{2}\left({ }^{1} \mathrm{H}\right.$ NMR), which converts to $8 \mathbf{a}$ in minutes. H/D exchange of N1-H with solvent is rapid relative to complexation, but exchange of $\mathrm{C} 3-\mathrm{H}$ may occur before or after $\pi$ complexation.21 Although the participation of platinum in the H/D exchange cannot be excluded, this exchange is rapid in the presence of 0.2 mole percent $\mathrm{BF}_{3}$-TFE solution (not containing 1a) and is complete in minutes at room temperature. NMR spectra are consistent with assignment of the $3 D$ tautomer $\mathbf{8}$ : in all three complexes $(\mathbf{8 a}, \mathbf{8 b}, \mathbf{8 c})$ the ${ }^{13} \mathrm{C}$ signal for the $\mathrm{C} 3$ methylene carbon appears as a multiplet at $\sim 44 \mathrm{ppm}$, and the ${ }^{1} \mathrm{H}$ signal for the $\mathrm{C} 2$ hydrogen appears as a singlet with ${ }^{195} \mathrm{Pt}$ satellites $\left(J_{\mathrm{Pt}-\mathrm{H}} \sim 40 \mathrm{~Hz}\right)$ at $\sim 8 \mathrm{ppm}$.

Reactions of methyl-substituted indoles were studied with cation 2a. Reactions of 2a with 1methylindole and 2-methylindole proceed through the intermediacy of observable and more stable $\pi$ complexes $\left(\mathbf{9}, \mathbf{1 1}\right.$; Scheme 6). ${ }^{22}{ }^{1} \mathrm{H}$ NMR spectra for $\mathbf{9}$ and $\mathbf{1 1}$ share important 
features with indene complex 3a: each shows a significantly upfield Pt- $\mathrm{CH}_{3}$ chemical shift relative to cation 2a. Upon rearrangement to the corresponding $N$-bound complexes $\mathbf{1 0}$ and 12, the Pt- $\mathrm{CH}_{3}$ signal returns downfield (Table 2). Other heterocycles (pyrrole, carbazole, benzofuran) did not afford clean results under these conditions; most notably, $N$-protected indoles gave rise to complex mixtures of products. 23

The mechanism of conversion of $\pi$-bound indole complexes to final $N$-bound complexes $\mathbf{8}$, 10, and 12 likely involves direct (intramolecular) slippage of platinum from the $\mathrm{C} 2=\mathrm{C} 3 \pi$ bond to $\mathrm{N} 1$ because the rate of $\pi$ to $\mathrm{N} 1$ conversion is independent of [indole]. Moreover, in a simple crossover experiment, a solution of the 2-methyl indole adduct 9 ( $\sim 90 \%$ conversion from 2a) was treated with 1 equivalent of 1-methylindole. Upon further reaction a distribution of $81(1) \% \mathbf{9}, 10(1) \% \mathbf{1 0}, 9(1) \% \mathbf{1 1}$ was observed, and finally a mixture containing $91(1) \% \mathbf{1 0}$ and $9(1) \% \mathbf{1 2}$ was observed. Thus little crossover is observed, excluding a mechanism involving indole displacement from the metal center.

Rearrangements of complexes $\mathbf{9}$ and $\mathbf{1 1}$ to complexes $\mathbf{1 0}$ and $\mathbf{1 2}$ are spontaneous at room temperature (also zero-order in indole). For the conversion of $\mathbf{2 a}$ to 10 at $30^{\circ} \mathrm{C}$, the rate of rearrangement of $\mathbf{9}$ to $\mathbf{1 0}$ is close to the rate for the binding of 2-methylindole to cation 2a, thus enabling NMR observation of all three species simultaneously in a single experiment (Figure 2). First order rate constants for the rearrangements of $\pi$ complexes $\mathbf{9}$ and $\mathbf{1 1}$ were measured at $40{ }^{\circ} \mathrm{C}$ in the presence of excess indole (Table 3). Strikingly, rearrangement of these $\pi$ complexes requires hours at $40{ }^{\circ} \mathrm{C}$, whereas $N$-ligated complexes 8 are fully formed at room temperature in only minutes, indicating that methylation of the indole in the 1 or 2 position substantially retards the rate of rearrangement of indole $\pi$ complexes.

The rate of rearrangement of indole $\pi$ complex $\mathbf{6 a}$ to $N$-ligated complex $8 \mathbf{a}$ is significantly diminished by added acid. When $\mathbf{2 a}$ is mixed with one equivalent of indole in the presence of a small amount ( 15 mole percent) of excess acid at room temperature, $\mathbf{6 a}$ is completely converted to 8a in minutes at room temperature, but conversion takes hours in the presence of 50 mole percent excess acid. In a more systematic set of experiments, 2a is prepared from 1a with a variable excess of acid, then five equivalents of indole is added, and reaction progress monitored by NMR at $40{ }^{\circ} \mathrm{C}$; apparent rate constants for rearrangement of $\mathbf{6 a}$ are shown in Table 4. Only modest reductions of rate are observed up to about three-fold excess acid, but when the excess acid becomes comparable to the amount of excess indole the rate slows dramatically (entry 4). Under these conditions indole complexation to form $\mathbf{6 a}$ is also slowed, to only approximately three times the rate of rearrangement (compared to at least two orders of magnitude faster in the absence of acid). Furthermore, the initial product of $\pi$ to N1 rearrangement is not $\mathbf{8 a}$ but a different $N$-adduct (presumably 7a); the rate of tautomerization of the first-formed $N$-adduct to the ultimate product $8 \mathbf{a}$ is slow under these acidic conditions.24 Thus protonation of the nitrogen lone pair slows both coordination of platinum to the $\mathrm{C}=\mathrm{C}$ double bond and subsequent migration of platinum to nitrogen; the inhibition of [1D] to [3D] indole tautomerization suggests that it is mediated by free (unprotonated) indole. 


\section{Conclusions}

The new indole complexes generated by addition of indole, 1-methylindole and 2methylindole to [(diimine) $\mathrm{Pt}(\mathrm{Me})(\mathrm{solv})]^{+}$cations are very rare examples of stable complexes of this class of heterocycle with late transition metals. The initial interaction is not with the nitrogen lone pair, but rather indole $\mathrm{C} 2=\mathrm{C} 3$ olefin ligation, the first examples of such complexation. Indene forms a more stable $\pi$ complex with cation 2a that undergoes subsequent activation of the indenyl $\mathrm{C}$ - $\mathrm{H}$ group in hours at $60{ }^{\circ} \mathrm{C}$ to afford methane and an $\eta 3$-indenyl adduct. By contrast, indole $\pi$ complexes of cation 2a rearrange to $\mathrm{N}$-ligated species. The rate of rearrangement can be controlled by substitution of the indole or by added $\mathrm{H}^{+}$. Presumably, productive $\mathrm{C}-\mathrm{H}$ activation chemistry on the indole nucleus is more likely to proceed from a complex of the indole $\mathrm{C} 2=\mathrm{C} 3$ olefin rather than $\mathrm{N} 1$; therefore strategies to prevent the formation of apparently inert $N$ complexes from the corresponding $\pi$ complexes are of interest to the continued development of synthetic strategies for functionalization of indoles by metal-mediated $\mathrm{C}-\mathrm{H}$ activation, although we have not yet achieved the latter. Progress toward these objectives is ongoing in our laboratories.

\section{Experimental Section}

\section{General Considerations}

${ }^{1} \mathrm{H}$ NMR and ${ }^{13} \mathrm{C}$ NMR spectra were recorded at ambient temperature using a Varian Inova 500,600 , or Mercury 300 spectrometer. The data are reported by chemical shift (ppm) from tetramethylsilane, multiplicity ( $\mathrm{s}$, singlet; $\mathrm{d}$, doublet; $\mathrm{t}$, triplet; $\mathrm{m}$, multiplet; dd, double doublet; dt, double triplet), coupling constants $(\mathrm{Hz})$, and integration. All ${ }^{13} \mathrm{C}$ NMR data were collected proton-decoupled $\left({ }^{13} \mathrm{C}\left\{{ }^{1} \mathrm{H}\right\}\right)$, except for those for $\mathbf{4 a}$, as specified. Mass spectra were acquired on a Finnigan LCQ ion trap or Agilent 5973 Network mass selective detector and were obtained by peak matching. All reactions were carried out under an atmosphere of nitrogen in glassware that had been oven-dried. Unless otherwise noted, all reagents were commercially obtained and, where appropriate, purified prior to use.

Tris(pentafluorophenyl)borane $\left[\mathrm{B}\left(\mathrm{C}_{6} \mathrm{~F}_{5}\right)_{3}\right]$ was purified by sublimation $\left(90^{\circ} \mathrm{C}, 0.5\right.$ Torr). 2,2,2-Trifluoroethanol- $d_{3}$ (TFE- $d_{3}$ ) was dried over 3 Å molecular sieves for at least 5 days and then vacuum distilled onto $\mathrm{B}\left(\mathrm{C}_{6} \mathrm{~F}_{5}\right)_{3}$. After $6 \mathrm{~h}$, the trifluoroethanol- $d_{3}$ was vacuum distilled and stored in a Teflon needle-valved vessel. Boron trifluoride was purified according to Brown's procedure 25 and stored as a solution in TFE- $d_{3}$. The platinum dimethyl complexes were synthesized following earlier reported procedures, as noted. 2,2,2Trifluoroethanol- $d_{3}, \mathrm{~B}\left(\mathrm{C}_{6} \mathrm{~F}_{5}\right)_{3}$, and platinum dimethyl complexes were stored in a Vacuum Atmospheres dinitrogen atmosphere drybox.

\section{Indene complex 3a}

Platinum dimethyl complex 1a4a $(19.2 \mu \mathrm{mol}, 10.5 \mathrm{mg})$ was weighed out in an oven-dried 4 $\mathrm{mL}$ vial in the drybox. TFE- $d_{3}(700 \mu \mathrm{L})$ and $\mathrm{BF}_{3}\left(0.477 \mathrm{M}\right.$ in TFE- $\left.d_{3}, 18 \mu \mathrm{mol}, 38 \mu \mathrm{L}\right)$ were then added and the suspension was stirred until it became a homogeneous orange solution. Indene (19.2 $\mu \mathrm{mol}, 2.24 \mathrm{mg}, 2.25 \mu \mathrm{L})$ was added, and the solution was transferred to an oven-dried J-Young NMR tube. After incubation at room temperature (24 hours in this case) the solution was analyzed by NMR and found to contain $\mathbf{3 a}$ in $>95 \%$ yield. 
${ }^{1} \mathrm{H}$ NMR $\left(600 \mathrm{MHz}, \mathrm{TFE}-d_{3}\right) \delta: 7.51\left(\mathrm{dt}, J_{\mathrm{H}-\mathrm{H}}=6.8 \mathrm{~Hz}\right.$ (doublet), $1.5 \mathrm{~Hz}$ (triplet), $\left.1 \mathrm{H}\right)$, 7.26-7.23 (m, 3H), $7.15(\mathrm{~s}, 1 \mathrm{H}), 7.10(\mathrm{~s}, 1 \mathrm{H}), 6.99(\mathrm{~s}, 1 \mathrm{H}), 6.96(\mathrm{~s}, 1 \mathrm{H}), 6.16\left(\mathrm{~d}, J_{\mathrm{H}-\mathrm{H}}=4\right.$ $\left.\mathrm{Hz}, J_{\mathrm{Pt}-\mathrm{H}}=79 \mathrm{~Hz}, 1 \mathrm{H}\right), 5.24\left(\right.$ apparent t $\left., J_{\mathrm{H}-\mathrm{H}} \sim 6.4 \mathrm{~Hz}, J_{\mathrm{Pt}-\mathrm{H}}=74 \mathrm{~Hz}, 1 \mathrm{H}\right), 3.30\left(\mathrm{~d}, J_{\mathrm{H}-\mathrm{H}}=\right.$ $\left.23 \mathrm{~Hz}, J_{\mathrm{Pt}-\mathrm{H}}=41 \mathrm{~Hz}, 1 \mathrm{H}\right), 3.07\left(\mathrm{~d}, J_{\mathrm{H}-\mathrm{H}}=23 \mathrm{~Hz}, J_{\mathrm{Pt}-\mathrm{H}}=38 \mathrm{~Hz}, 1 \mathrm{H}\right), 2.32(\mathrm{~s}, 6 \mathrm{H}), 2.25(\mathrm{~s}$, $3 \mathrm{H}), 2.20(\mathrm{~s}, 3 \mathrm{H}), 2.13(\mathrm{~s}, 3 \mathrm{H}), 2.12(\mathrm{~s}, 3 \mathrm{H}), 2.07(\mathrm{~s}, 3 \mathrm{H}), 1.97(\mathrm{~s}, 3 \mathrm{H}),-0.74\left(\mathrm{~s}, J_{\mathrm{Pt}-\mathrm{H}}=53\right.$ $\mathrm{Hz}, 3 \mathrm{H}) .{ }^{13} \mathrm{C}$ NMR $\left(150 \mathrm{MHz}, \mathrm{TFE}-d_{3}\right) \delta: 185.6,178.7,148.4,142.7,141.5,141.0,140.9$, 139.7, 131.8, 131.6, 131.0, 131.0, 130.8, 130.5, 130.4, 129.5, 129.2, 128.9, 126.2, 125.5, 103.1, 92.4, 40.7, 21.1, 21.1, 20.4, 20.2, 17.9, 17.9, 17.9, 17.8, 4.7. FTIR (neat): $2920(\mathrm{w})$, 1476 (w), 1384 (w), 1237 (w), 1180 (m), 1160 (m), 1051 (s), 847 (w), $766(w), 718$ (w) $\mathrm{cm}^{-1}$. $\mathrm{FAB}^{+} \mathrm{MS}$, calcd for $\mathrm{C}_{32} \mathrm{H}_{40} \mathrm{~N}_{2} \mathrm{Pt}\left([\mathrm{M}+\mathrm{H}]^{+}\right)$: 647.2840, found 647.2822. Calcd for $\mathrm{C}_{31} \mathrm{H}_{36} \mathrm{~N}_{2} \mathrm{Pt}\left([\mathrm{M}-\mathrm{Me}]^{+}\right)$: 631.2526, found 631.2302.

Indene complex 3a- $d_{3}$ was prepared as above. ${ }^{1} \mathrm{H}$ NMR $\left(500 \mathrm{MHz}\right.$, TFE- $\left.d_{3}\right)$ matches the data above, except that signals at $\delta=6.16,3.30$, and $3.07 \mathrm{ppm}$ are absent.

\section{Indene complexes $3 b, 3 c, 3 d$}

Complexes $\mathbf{3 b}, \mathbf{3 c}, \mathbf{3 d}$ were prepared by direct analogy to complex $\mathbf{3 a}$ above, starting from platinum dimethyl species 1b, 1d4a and 1c. 3 3b: ${ }^{1} \mathrm{H}$ NMR (300 MHz, TFE- $\left.d_{3}\right) \delta: 7.51(\mathrm{~d}$, $7.23 \mathrm{~Hz}, 1 \mathrm{H}$ ), 7.29-7.19 (m, 3H), 7.02 (broad s, 2H), 6.57 (broad s, 4H), 6.46 (broad s, 1H), 5.64 (broad s, 1H), 3.20 (broad s, 2H), 2.32 (broad s, 12H), 2.07 (broad s, 6H), -0.59 (s, $\left.J_{\mathrm{Pt}-\mathrm{H}}=71 \mathrm{~Hz}, 3 \mathrm{H}\right) .3 \mathrm{c}:{ }^{1} \mathrm{H}$ NMR $\left(300 \mathrm{MHz}, \mathrm{TFE}-d_{3}\right) \delta: 7.96(\mathrm{~s}, 2 \mathrm{H}), 7.56-7.52(\mathrm{~m}, 1 \mathrm{H})$, $7.53(\mathrm{~s}, 4 \mathrm{H}), 7.29-7.11(\mathrm{~m}, 3 \mathrm{H}), 6.53(\mathrm{~s}, 1 \mathrm{H}), 5.68(\mathrm{~s}, 1 \mathrm{H}), 3.22(\mathrm{~s}, 2 \mathrm{H}), 2.12(\mathrm{~s}, 6 \mathrm{H}),-0.34$ $(\mathrm{s},=69 \mathrm{~Hz}, 3 \mathrm{H}) .3 \mathrm{~d}:{ }^{1} \mathrm{H}$ NMR $\left(300 \mathrm{MHz}\right.$, TFE- $\left.J_{\mathrm{Pt}-\mathrm{H}} d_{3}\right) \delta: 7.52-7.47(\mathrm{~m}, 3 \mathrm{H}), 7.31-7.20(\mathrm{~m}$, 3H), 6.84 (broad s, 4H), 6.33 (broad s, 1H), 5.54 (broad s, 1H), 3.13 (broad s, 2H), 2.11 (broad s, 6H), 1.33 (broad s, 36H), $-0.66\left(\mathrm{~s}, J_{\mathrm{Pt}-\mathrm{H}}=69 \mathrm{~Hz}, 3 \mathrm{H}\right)$.

\section{Eyring Analysis for the conversion of $3 a$ to $4 a$}

3a was prepared as a solution in TFE- $d_{3}$ as described above, with the exception that $2.50 \mathrm{eq}$ of $\mathrm{B}\left(\mathrm{C}_{6} \mathrm{~F}_{5}\right)_{3}$ was used as the acid source. Conversion and rate were then recorded as described in the main text.

\section{Indenyl complex 4a}

The solution of 3a prepared above was heated in its J-Young tube to the temperature and time described in the main text. ${ }^{1} \mathrm{H}$ NMR (500 MHz, TFE- $\left.d_{3}\right) \delta: 7.12-7.10(\mathrm{~m}, 6 \mathrm{H}), 6.79$ (dd $\left.J_{\mathrm{H}-\mathrm{H}}=5.7,3.1 \mathrm{~Hz}, \sim 3 \mathrm{H}\right), 5.01(\mathrm{~s}, 262 \mathrm{H}), 2.40(\mathrm{~s}, 6 \mathrm{H}), 2.12(\mathrm{~s}, 6 \mathrm{H}), 1.97(\mathrm{~s}, 12 \mathrm{H}) .{ }^{13} \mathrm{C} \mathrm{NMR}$ $\left(150 \mathrm{MHz}, \mathrm{TFE}-d_{3}\right) \delta: 172.6(2 \mathrm{C}), 147.6(2 \mathrm{C}), 140.8(2 \mathrm{C}), 134.6,131.2(4 \mathrm{C}), 129.9(4 \mathrm{C})$, $129.3(4 \mathrm{C}), 120.0(2 \mathrm{C}), 71.6\left(J_{\mathrm{Pt}-\mathrm{C}}=130 \mathrm{~Hz}, 2 \mathrm{C}\right), 21.2(2 \mathrm{C}), 18.0(2 \mathrm{C}), 17.6(4 \mathrm{C}) .{ }^{13} \mathrm{C}$ $\operatorname{NMR}\left(125 \mathrm{MHz}\right.$, not $\{1 \mathrm{H}\}$, TFE- $\left.d_{3}\right) \delta: 172.5(\mathrm{~s}, 2 \mathrm{C}), 147.6(\mathrm{~s}, 2 \mathrm{C}), 140.8\left(\mathrm{~d}, J_{\mathrm{C}-\mathrm{H}}=6 \mathrm{~Hz}\right.$, 2C), 134.6, 132-130 (m, $\left.J_{1, \mathrm{C}-\mathrm{H}} \sim 150 \mathrm{~Hz}, 2 \mathrm{C}\right), 131.2\left(\mathrm{~d}, J_{\mathrm{C}-\mathrm{H}}=152 \mathrm{~Hz}, 2 \mathrm{C}\right), 129.9\left(\mathrm{~d}, J_{\mathrm{C}-\mathrm{H}}\right.$ $=154 \mathrm{~Hz}, 4 \mathrm{C}), 129.3(\mathrm{~s}, 4 \mathrm{C}), 120.0\left(\mathrm{~d}, J_{\mathrm{C}-\mathrm{H}}=163 \mathrm{~Hz}, 2 \mathrm{C}\right), 71.6\left(\mathrm{~d}, J_{\mathrm{C}-\mathrm{H}}=181 \mathrm{~Hz}, 2 \mathrm{C}\right)$, $21.2\left(\mathrm{q}, J_{\mathrm{CH}}=126 \mathrm{~Hz}, 2 \mathrm{C}\right), 18.0\left(\mathrm{q}, J_{\mathrm{C}-\mathrm{H}}=132 \mathrm{~Hz}, 2 \mathrm{C}\right), 17.6\left(\mathrm{q}, J_{\mathrm{C}-\mathrm{H}}=126 \mathrm{~Hz}, 4 \mathrm{C}\right)$. FTIR (neat): 2919 (w), 1476 (w), 1331 (w), 1385, 1244 (w), 1182 (m), 1157 (m), 1054 (s), 851, $754(\mathrm{w}) \mathrm{cm}^{-1}$. $\mathrm{FAB}^{+} \mathrm{MS}$, calcd for $\mathrm{C}_{31} \mathrm{H}_{36} \mathrm{~N}_{2} \mathrm{Pt}\left([\mathrm{M}+\mathrm{H}]^{+}\right): 631.2526$, found $631.25301 .{ }^{2} \mathrm{H}$ NMR $\left(76.7 \mathrm{MHz}, \mathrm{TFE}-h_{3}\right)$ for this compound is shown in the Supporting Information. 
Indenyl complex 4a- $d_{2}$ was prepared as above. ${ }^{1} \mathrm{H}$ NMR (500 MHz, TFE- $\left.d_{3}\right)$ matches the data above, except that the signal at $\delta=5.01 \mathrm{ppm}$ is absent. $\mathrm{FAB}^{+} \mathrm{MS}$, calcd for $\mathrm{C}_{31} \mathrm{H}_{34} \mathrm{D}_{2} \mathrm{~N}_{2} \mathrm{Pt}\left(\left[\mathrm{M}-d_{2}+\mathrm{H}\right]^{+}\right): 633.2652$, and for $\mathrm{C}_{31} \mathrm{H}_{32} \mathrm{D}_{3} \mathrm{~N}_{2} \mathrm{Pt}\left(\left[\mathrm{M}-d_{3}\right]^{+}\right): 633.2637$; found 33.2631. ${ }^{2} \mathrm{H}$ NMR (76.7 MHz, TFE- $h_{3}$ ) for this compound is shown in the Supporting Information.

\section{Indole $\pi$-complex $6 \mathbf{a}$}

Complex 6a is observed as an intermediate in kinetic runs (Table 4, entry 4). ${ }^{1} \mathrm{H}$ NMR (500 MHz, TFE- $\left.d_{3}\right) \delta: 7.62(\mathrm{~s}, 1 \mathrm{H}), 7.60\left(\mathrm{~d}, J_{\mathrm{H}-\mathrm{H}}=7.8 \mathrm{~Hz}, 1 \mathrm{H}\right), 7.25-7.22(\mathrm{~m}, 2 \mathrm{H}), 7.17\left(\mathrm{t}, J_{\mathrm{H}-\mathrm{H}}\right.$ $=8.0 \mathrm{~Hz}, 1 \mathrm{H}), 7.14(\mathrm{~s}, 1 \mathrm{H}), 7.01(\mathrm{~s}, 1 \mathrm{H}), 6.97(\mathrm{~s}, 1 \mathrm{H}), 6.94(\mathrm{~s}, 1 \mathrm{H}), 5.40\left(\mathrm{~s}, J_{\mathrm{H}-\mathrm{H}}=94 \mathrm{~Hz}\right.$, $\sim 0 \mathrm{H}), 272.39(\mathrm{~s}, 3 \mathrm{H}), 2.31(\mathrm{~s}, 3 \mathrm{H}), 2.25(\mathrm{~s}, 3 \mathrm{H}), 2.18(\mathrm{~s}, 3 \mathrm{H}), 2.13(\mathrm{~s}, 3 \mathrm{H}), 2.03(\mathrm{~s}, 3 \mathrm{H}), 1.83$ $(\mathrm{s}, 3 \mathrm{H}), 1.81(\mathrm{~s}, 3 \mathrm{H}),-1.02\left(\mathrm{~s}, J_{\mathrm{Pt}-\mathrm{H}}=62 \mathrm{~Hz}, 3 \mathrm{H}\right)$.

\section{Indole $\mathbf{N}$-complex 8a}

Platinum dimethyl complex 1a $4 \mathrm{a}(46.6 \mu \mathrm{mol}, 25.4 \mathrm{mg})$ was weighed out in an oven-dried 4 $\mathrm{mL}$ vial in the drybox. TFE- $d_{3}(350 \mu \mathrm{L})$ and $\mathrm{BF}_{3}\left(0.455 \mathrm{M}\right.$ in TFE- $\left.d_{3}, 53.5 \mu \mathrm{mol}, 118 \mu \mathrm{L}\right)$ were then added and the suspension was stirred until it became a homogeneous orange solution. Indole ( $47 \mu \mathrm{mol}, 5.5 \mathrm{mg}$ ) was added, and the solution was transferred, rinsing with TFE- $d_{3}(350 \mu \mathrm{L})$ to an oven-dried J-Young NMR tube. The solution was analyzed by NMR and found to contain $8 \mathbf{a}$ in $>95 \%$ yield. ${ }^{1} \mathrm{H}$ NMR $\left(500 \mathrm{MHz}\right.$, TFE- $\left.d_{3}\right) \delta: 7.89\left(\mathrm{~s}, J_{\mathrm{Pt}-\mathrm{H}}=34\right.$ $\mathrm{Hz}, 1 \mathrm{H}), 7.73\left(\mathrm{~d}, J_{\mathrm{H}-\mathrm{H}}=7.3 \mathrm{~Hz}, 1 \mathrm{H}\right), 7.40-7.35(\mathrm{~m}, 3 \mathrm{H}), 7.12(\mathrm{~s}, 1 \mathrm{H}), 7.09(\mathrm{~s}, 1 \mathrm{H}), 6.97(\mathrm{~s}$, $1 \mathrm{H}), 6.50(\mathrm{~s}, 1 \mathrm{H}), 2.35(\mathrm{~s}, 3 \mathrm{H}), 2.34(\mathrm{~s}, 3 \mathrm{H}), 2.27(\mathrm{~s}, 3 \mathrm{H}), 2.20(\mathrm{~s}, 3 \mathrm{H}), 2.16(\mathrm{~s}, 3 \mathrm{H}), 1.88(\mathrm{~s}$, $3 \mathrm{H}), 1.87(\mathrm{~s}, 3 \mathrm{H}), 1.80(\mathrm{~s}, 3 \mathrm{H}), 0.61\left(\mathrm{~s}, J_{\mathrm{Pt}-\mathrm{H}}=73 \mathrm{~Hz}, 3 \mathrm{H}\right) .{ }^{13} \mathrm{C}$ NMR $\left(125 \mathrm{MHz}, \mathrm{TFE}-d_{3}\right) \delta$ : 182.1, 175.6, 175.1, 153.9, 142.7, 140.9, 140.4, 139.8, 134.5, 130.9, 130.9, 130.8, 130.7, 130.6, 130.5, 130.1, 130.0, 129.3, 128.9, 125.8, 122.3, 44 (m, 1C), 21.1, 20.8, 20.0, 19.5, 17.9, 17.8 (2C), 17.7, -12.1. FTIR (neat): 2921 (m), $2094(\mathrm{w}), 1474(\mathrm{~m}), 1449(\mathrm{~m}), 1382$ (m), $1313(\mathrm{~m}), 1240(\mathrm{~m}), 1184$ (s), 1128 (s), 1069 (s), 1018 (s), 852 (m), 818 (m), 769 (m), $644(\mathrm{~m}), 534(\mathrm{~m}) \mathrm{cm}^{-1}$. FAB ${ }^{+} \mathrm{MS}$, calc'd for $\mathrm{C}_{31} \mathrm{H}_{35} \mathrm{~N}_{3} \mathrm{Pt}^{2} \mathrm{H}_{2}$ ([M-H] $\left.]^{+}\right): 649.2839$, found 649.2847 .

\section{Indole complex 8b}

Platinum dimethyl complex 1b4a (32.5 $\mu \mathrm{mol}, 16.8 \mathrm{mg})$ was weighed out in an oven-dried 4 $\mathrm{mL}$ vial in the drybox. TFE- $d_{3}(700 \mu \mathrm{L})$ and $\mathrm{BF}_{3}\left(0.478 \mathrm{M}\right.$ in TFE- $\left.d_{3}, 37.3 \mu \mathrm{mol}, 78.1 \mu \mathrm{L}\right)$ were then added and the suspension was stirred until it became a homogeneous orange solution. Indole ( $33 \mu \mathrm{mol}, 3.8 \mathrm{mg}$ ) was added, and the solution was transferred to an ovendried screw-capped NMR tube. The solution was analyzed by NMR and found to contain $\mathbf{8 b}$ in $>95 \%$ yield.

${ }^{1} \mathrm{H}$ NMR $\left(500 \mathrm{MHz}, \mathrm{TFE}-d_{3}\right) \delta: 8.01\left(\mathrm{~s}, J_{\mathrm{Pt}-\mathrm{H}}=36 \mathrm{~Hz}, 1 \mathrm{H}\right), 7.87\left(\mathrm{~d}, J_{\mathrm{H}-\mathrm{H}}=7.9 \mathrm{~Hz}, 1 \mathrm{H}\right)$, 7.46-7.41 (m, 2H), 7.38-7.35 (m, 1H), 7.06 (s, 1H), $6.75(\mathrm{~s}, 1 \mathrm{H}), 6.71(\mathrm{~s}, 1 \mathrm{H}), 6.66(\mathrm{~s}, 1 \mathrm{H})$, 6.49 (s, broad, 1H), 6.06 (s, broad, 1H), 2.39 (s, 3H), 2.37 (s, 3H), 2.25 (s, broad, 3H), 1.96 (s, 3H), 1.95 (s, 3H), 1.69 (s, 3H), 0.74 (s, $\left.J_{\mathrm{Pt}-\mathrm{H}}=73 \mathrm{~Hz}, 3 \mathrm{H}\right) .{ }^{13} \mathrm{C}$ NMR $(125 \mathrm{MHz}$, TFE$\left.d_{3}\right) \delta: 182.3,174.6,175.1,153.9,147.8,145.7,141.9,141.8,141.9-141.5$ (m, broad, 2C), 134.5, 131.2, 130.5, 130.2, 129.4, 125.9, 122.7, 121.0, 121.0, 120.7 (broad, 1C), 119.5 (broad, 1C), 44 (m, 1C), 21.6, 21.5, 21.2, 21.9-21.8 (m, 2C), -11.3. FTIR (neat): 3014 (w), 
$2921(w), 2875$ (w), 1609 (w), 1593 (w), 1468 (w), 1453 (w), 1384 (w), 1308 (w), $1185(\mathrm{~m})$, $1130(\mathrm{~m}), 1060(\mathrm{~s}), 863(\mathrm{w}), 771(\mathrm{w}), 685(\mathrm{w}) \mathrm{cm}^{-1}$. FAB ${ }^{+} \mathrm{MS}$, calcd for $\mathrm{C}_{29} \mathrm{H}_{31} \mathrm{~N}_{3} \mathrm{Pt}^{2} \mathrm{H}_{2}$ $\left([\mathrm{M}-\mathrm{H}]^{+}\right): 620.2448$, found 620.2459 .

\section{Indole complex 8c}

Platinum dimethyl complex 1c3 $(36.0 \mu \mathrm{mol}, 26.4 \mathrm{mg})$ was weighed out in an oven-dried 4 $\mathrm{mL}$ vial in the drybox. TFE- $d_{3}(700 \mu \mathrm{L})$ and $\mathrm{BF}_{3}\left(0.478 \mathrm{M}\right.$ in TFE- $\left.d_{3}, 41.4 \mu \mathrm{mol}, 86.6 \mu \mathrm{L}\right)$ were then added and the suspension was stirred until it became a homogeneous orange solution. Indole ( $36 \mu \mathrm{mol}, 9.2 \mathrm{mg}$ ) was added, and the solution was transferred to an ovendried screw-capped NMR tube. The solution was analyzed by NMR and found to contain $\mathbf{8 c}$ in $>95 \%$ yield.

${ }^{1} \mathrm{H}$ NMR $\left(500 \mathrm{MHz}, \mathrm{TFE}-d_{3}\right) \delta: 8.27\left(\mathrm{~s}, J_{\mathrm{Pt}-\mathrm{H}} \sim 40 \mathrm{~Hz}, 1 \mathrm{H}\right), 8.02(\mathrm{~s}, 1 \mathrm{H}), 7.78\left(\mathrm{~d}, J_{\mathrm{H}-\mathrm{H}}=8.5\right.$ $\mathrm{Hz}, 1 \mathrm{H}), 7.73$ (s, 1H), $7.68(\mathrm{~s}, 1 \mathrm{H}), 7.55(\mathrm{~S}, 1 \mathrm{H}), 7.45-7.27$ (m, 5H), $2.04(\mathrm{~s}, 3 \mathrm{H}), 2.03(\mathrm{~s}$, $3 \mathrm{H}), 0.76\left(\mathrm{~s}, J_{\mathrm{Pt}-\mathrm{H}} \sim 60 \mathrm{~Hz}, 3 \mathrm{H}\right) .{ }^{13} \mathrm{C}$ NMR $\left(125 \mathrm{MHz}, \mathrm{TFE}-d_{3}\right) \delta: 184.5,177.7,175.6(\mathrm{~m}$, 1C), 152.9 (m, 1C), 148.5, 147.0, 136.1-134.1 (m, apparent 4C), 134.1, 130.7, 129.4, 126.2, 124.6 (apparent 2C), 124-122 (m, apparent 4C), $122.3\left(\mathrm{q}, J_{\mathrm{C}-\mathrm{F}}=227 \mathrm{~Hz}\right), 122.0, \sim 44(\mathrm{~m}$, 1C), 21.9, 20.5, -9.4. Three $\mathrm{CF}_{3}$ quartets cannot be identified because of coincidences with the solvent $\mathrm{CF}_{3}$; each has $\delta \sim 126 \mathrm{ppm}, J_{\mathrm{C}-\mathrm{F}} \sim 280 \mathrm{~Hz}$. FTIR (neat): 3065 (w), $2932(\mathrm{w})$, $2882(\mathrm{w}), 1622$ (w), 1460 (m), 1373 (s), 1281 (s), 1182 (s), 1141 (s), 1067 (m), 903 (m), 848 (w), 771 (w), 705 (w), $684(\mathrm{~m}) \mathrm{cm}^{-1}$. FAB ${ }^{+} \mathrm{MS}$, calcd for $\mathrm{C}_{29} \mathrm{H}_{19} \mathrm{~F}_{12} \mathrm{~N}_{3} \mathrm{Pt}^{2} \mathrm{H}_{2}\left([\mathrm{M}-\mathrm{H}]^{+}\right)$: 836.1317, found 836.1333.

\section{2-Methylindole- $\pi$-complex 9}

Platinum dimethyl complex 1a4a $(33.4 \mu \mathrm{mol}, 18.2 \mathrm{mg})$ was weighed out in an oven-dried 4 $\mathrm{mL}$ vial in the drybox. TFE- $d_{3}(700 \mu \mathrm{L})$ and $\mathrm{BF}_{3}\left(0.455 \mathrm{M}\right.$ in TFE- $\left.d_{3}, 38.4 \mu \mathrm{mol}, 84.3 \mu \mathrm{L}\right)$ were then added and the suspension was stirred until it became a homogeneous orange solution. 2-Methylindole ( $33 \mu \mathrm{mol}, 4.4 \mathrm{mg}$ ) was added, and the solution was transferred to an oven-dried screw-capped NMR tube. The solution was analyzed by NMR and found to contain 9 in $>95 \%$ yield.

${ }^{1} \mathrm{H}$ NMR $\left(500 \mathrm{MHz}, \mathrm{TFE}-d_{3}\right) \delta: 7.35\left(\mathrm{~d}, J_{\mathrm{H}-\mathrm{H}}=7.5 \mathrm{~Hz}, 1 \mathrm{H}\right), 7.29\left(\mathrm{~d}, J_{\mathrm{H}-\mathrm{H}}=7.5 \mathrm{~Hz}, 1 \mathrm{H}\right)$, $7.25(\mathrm{~s}, 1 \mathrm{H}), 7.20-7.14(\mathrm{~m}, 3 \mathrm{H}), 6.97(\mathrm{~s}, 1 \mathrm{H}), 6.90(\mathrm{~s}, 1 \mathrm{H}), 2.53(\mathrm{~s}, 3 \mathrm{H}), 2.43(\mathrm{~s}, 3 \mathrm{H}), 2.35(\mathrm{~s}$, $3 \mathrm{H}), 2.29(\mathrm{~s}, 3 \mathrm{H}), 2.25(\mathrm{~s}, 3 \mathrm{H}), 2.10(\mathrm{~s}, 3 \mathrm{H}), 1.90(\mathrm{~s}, 3 \mathrm{H}), 1.83(\mathrm{~s}, 3 \mathrm{H}), 1.63(\mathrm{~s}, 3 \mathrm{H}),-0.75$ $\left(\mathrm{s}, J_{\mathrm{Pt}-\mathrm{H}}=67,3 \mathrm{H}\right) .{ }^{13} \mathrm{C}$ NMR $\left(125 \mathrm{MHz}\right.$, TFE- $\left.d_{3}\right) \delta: 178.0,176.8,143.3,142.7,141.9$, $141.5,140.2,139.7,131.5,131.4,130.7,130.6,130.5,130.4,130.1,129.4,126.7,126.1$, $123.9,114.1,21.1,21.0,20.2,19.9,18.1,18.0,17.5$ (2C), -3.6. (3 C cannot be uniquely identified because of dispersity in the aryl region and the transitory nature of 9.) FTIR (neat): 2918 (w), 1612 (w), 1505 (w), 1479 (w), 1458 (w), 1383 (w), 1329 (w), 1238 (m), $1157(\mathrm{~m}), 1059(\mathrm{~s}), 848(\mathrm{w}), 742(\mathrm{~m}), 571(\mathrm{w}), 534(\mathrm{w}) \mathrm{cm}^{-1} .9$ is not sufficiently long-lived for HRMS.

\section{2-Methylindole- $\mathrm{N}$-complex 10}

The crude solution of $\mathbf{9}$ prepared above was incubated at room temperature for 50 hours to give $\mathbf{1 0}$ in $>95 \%$ yield. 
${ }^{1} \mathrm{H}$ NMR $\left(500 \mathrm{MHz}, \mathrm{TFE}-d_{3}\right) \delta: 7.74\left(\mathrm{~d}, J_{\mathrm{H}-\mathrm{H}}=7.8 \mathrm{~Hz}, 1 \mathrm{H}\right), 7.32\left(\mathrm{~d}, J_{\mathrm{H}-\mathrm{H}}=7.9 \mathrm{~Hz}, 1 \mathrm{H}\right)$, 7.29-7.27 (m, 2H), $7.11(\mathrm{~s}, 1 \mathrm{H}), 7.10(\mathrm{~s}, 1 \mathrm{H}), 6.87(\mathrm{~s}, 1 \mathrm{H}), 6.44(\mathrm{~s}, 1 \mathrm{H}), 2.41(\mathrm{~s}, 3 \mathrm{H}), 2.35(\mathrm{~s}$, $3 \mathrm{H}), 2.33(\mathrm{~s}, 3 \mathrm{H}), 2.24(\mathrm{~s}, 3 \mathrm{H}), 2.19(\mathrm{~s}, 3 \mathrm{H}), 2.12(\mathrm{~s}, 3 \mathrm{H}), 1.86(\mathrm{~s}, 3 \mathrm{H}), 1.85(\mathrm{~s}, 3 \mathrm{H}), 1.83(\mathrm{~s}$, $3 \mathrm{H}), 0.53\left(\mathrm{~s}, J_{\mathrm{Pt}-\mathrm{H}}=74,3 \mathrm{H}\right) .{ }^{13} \mathrm{C}$ NMR $\left(125 \mathrm{MHz}\right.$, TFE- $\left.d_{3}\right) \delta: 186.0,181.7,175.8,154.9$, $142.8,142.0,140.3,139.6,135.0,130.9,130.7,130.4,129.9,128.9,128.8,128.7,122.5$, 121.6, 121.1, 121.0, 112.4, 45 (m, 1C), 22.1, 21.1, 20.8, 20.1, 19.5, 18.4, 17.9, 17.8, 13.5, -13.7. FTIR (neat): 3512 (broad, w), 3399 (broad, w), 2921 (broad, w), 1613 (w), 1573 (w), $1476(\mathrm{~m}), 1456(\mathrm{~m}), 1384(\mathrm{~m}), 1316(\mathrm{~m}), 1240(\mathrm{~m}), 1187(\mathrm{~s}), 1130(\mathrm{~s}), 1069(\mathrm{~s}), 852(\mathrm{w})$, $756(\mathrm{w}), 646(\mathrm{w}), 535(\mathrm{w}) \mathrm{cm}^{-1}$. $\mathrm{FAB}^{+} \mathrm{MS}$, calc'd for $\mathrm{C}_{32} \mathrm{H}_{38} \mathrm{~N}_{3} \mathrm{Pt}^{2} \mathrm{H}_{2}\left([\mathrm{M}]^{+}\right)$: 663.2996, found 663.2980 .

\section{1-Methylindole- $\pi$-complex 11}

Platinum dimethyl complex 1a4a ( $46.9 \mu \mathrm{mol}, 25.6 \mathrm{mg})$ was weighed out in an oven-dried 4 $\mathrm{mL}$ vial in the drybox. TFE- $d_{3}(700 \mu \mathrm{L})$ and $\mathrm{BF}_{3}\left(0.455 \mathrm{M}\right.$ in TFE- $\left.d_{3}, 54 \mu \mathrm{mol}, 119 \mu \mathrm{L}\right)$ were then added and the suspension was stirred until it became a homogeneous orange solution. 1-Methylindole ( $46.9 \mu \mathrm{mol}, 6.16 \mathrm{mg}, 6.00 \mu \mathrm{L}$ ) was added, and the solution was transferred to an oven-dried screw-capped NMR tube. The solution was analyzed by NMR and found to contain $\mathbf{1 1}$ in $>95 \%$ yield.

${ }^{1} \mathrm{H}$ NMR $\left(500 \mathrm{MHz}, \mathrm{TFE}-d_{3}\right) \delta: 7.57\left(\mathrm{~d}, J_{\mathrm{H}-\mathrm{H}}=8.7 \mathrm{~Hz}, 1 \mathrm{H}\right), 7.50\left(\mathrm{~s}, J_{\mathrm{Pt}-\mathrm{H}}=49 \mathrm{~Hz}, 1 \mathrm{H}\right)$, $7.28\left(\mathrm{t}, J_{\mathrm{H}-\mathrm{H}}=7.8 \mathrm{~Hz}, 1 \mathrm{H}\right), 7.22-7.17(\mathrm{~m}, 2 \mathrm{H}), 7.16(\mathrm{~s}, 1 \mathrm{H}), 7.07(\mathrm{~s}, 1 \mathrm{H}), 6.97(\mathrm{~s}, 1 \mathrm{H}), 6.91$ $(\mathrm{s}, 1 \mathrm{H}), 5.95\left(\mathrm{~s}, J_{\mathrm{H}-\mathrm{H}}=88 \mathrm{~Hz}, \sim 0 \mathrm{H}\right), 273.65(\mathrm{~s}, 3 \mathrm{H}), 2.41(\mathrm{~s}, 3 \mathrm{H}), 2.32(\mathrm{~s}, 3 \mathrm{H}), 2.24(\mathrm{~s}, 6 \mathrm{H})$, $2.14(\mathrm{~s}, 3 \mathrm{H}), 2.00(\mathrm{~s}, 3 \mathrm{H}), 1.86(\mathrm{~s}, 3 \mathrm{H}), 1.80(\mathrm{~s}, 3 \mathrm{H}),-1.16\left(\mathrm{~s}, J_{\mathrm{Pt}-\mathrm{H}}=70 \mathrm{~Hz}, 3 \mathrm{H}\right) .{ }^{13} \mathrm{C} \mathrm{NMR}$ $\left(125 \mathrm{MHz}\right.$, TFE- $\left.d_{3}\right) \delta: 179.4,177.1,144.9,142.6,141.5,140.4,140.1,137.3,131.7,131.5$, $130.8,130.7,130.6,130.2,130.1,129.8,125.2,125.0,128.9,128.3,111.9,34.2,21.1,21.0$, $20.0,19.8,18.0,17.7(2 \mathrm{C}), 17.6,-3.0$. Because of dispersion in the $130 \mathrm{ppm}$ region, $1 \mathrm{C}$ cannot be located. FTIR (neat): 2919 (w), 1477 (w), 1454 (w), 1383 (w), 1327 (w), 1238 (w), $1184(\mathrm{~m}), 1128(\mathrm{~m}), 1054(\mathrm{~s}), 847(\mathrm{w}), 753(\mathrm{w}), 679(\mathrm{w}) \mathrm{cm}^{-1}$. FAB ${ }^{+}$MS, calc'd for $\mathrm{C}_{32} \mathrm{H}_{39} \mathrm{~N}_{3} \mathrm{Pt}^{2} \mathrm{H}_{1}\left([\mathrm{M}]^{+}\right)$: 662.2933, found 662.2932.

\section{1-Methylindole- $\mathrm{N}$-complex 12}

The crude solution of $\mathbf{1 1}$ prepared above was incubated at room temperature for 2 days, then heated to $40{ }^{\circ} \mathrm{C}$ for 4 hours to give $\mathbf{1 2}$ in $\sim 78 \%$ yield by NMR.

${ }^{1} \mathrm{H}$ NMR $\left(500 \mathrm{MHz}, \mathrm{TFE}-d_{3}\right) \delta: 7.32\left(\mathrm{t}, J_{\mathrm{H}-\mathrm{H}}=7.6 \mathrm{~Hz}, 1 \mathrm{H}\right), 7.28\left(\mathrm{~d}, J_{\mathrm{H}-\mathrm{H}}=7.4 \mathrm{~Hz}, 1 \mathrm{H}\right)$, $7.22\left(\mathrm{t}, J_{\mathrm{H}-\mathrm{H}}=7.4 \mathrm{~Hz}, 1 \mathrm{H}\right), 7.19\left(\mathrm{~d}, J_{\mathrm{H}-\mathrm{H}}=8.4 \mathrm{~Hz}, 1 \mathrm{H}\right), 7.08(\mathrm{~s}, 1 \mathrm{H}), 7.07(\mathrm{~s}, 1 \mathrm{H}), 6.91(\mathrm{~s}$, 1H), $6.69(\mathrm{~s}, 1 \mathrm{H}), 3.83(\mathrm{~s}, 3 \mathrm{H}), 2.33(\mathrm{~s}, 3 \mathrm{H}), 2.30(\mathrm{~s}, 3 \mathrm{H}), 2.24(\mathrm{~s}, 3 \mathrm{H}), 2.18(\mathrm{~s}, 3 \mathrm{H}), 2.13(\mathrm{~s}$, $3 \mathrm{H}), 2.01(\mathrm{~s}, 3 \mathrm{H}), 1.98(\mathrm{~s}, 3 \mathrm{H}), 1.85(\mathrm{~s}, 3 \mathrm{H}), 0.26\left(\mathrm{~s}, J_{\mathrm{Pt}-\mathrm{H}}=77 \mathrm{~Hz}, 3 \mathrm{H}\right) .{ }^{13} \mathrm{C}$ NMR $(125$ MHz, TFE- $\left.d_{3}\right) \delta: 180.1,176.3,148.4,144.6,142.0,139.9,139.7,137.0,136.9,130.9,130.7$, $130.7,130.4,130.2,129.3,128.8,128.8,128.6,127.6,125.6,125.5,112.7,39.9\left(J_{\mathrm{Pt}-\mathrm{C}}=81\right.$ Hz), 21.1, 20.7, 20.0, 19.8, 17.9, 17.9, 17.9, 17.8, -16.8. FTIR (neat): 2923 (m, broad), 2100 (w), 1609 (w), $1515(\mathrm{~m}), 1463(\mathrm{~s}), 1384(\mathrm{~m}), 1312(\mathrm{~m}), 1238(\mathrm{~m}), 1186(\mathrm{~s}), 1130$ (s), 1069 (s, broad), $860(\mathrm{w}), 818(\mathrm{w}), 759(\mathrm{~m}), 646(\mathrm{w}), 535(\mathrm{w}) \mathrm{cm}^{-1}$. FAB ${ }^{+} \mathrm{MS}$, calcd for $\mathrm{C}_{32} \mathrm{H}_{39} \mathrm{~N}_{3} \mathrm{Pt}^{2} \mathrm{H}\left([\mathrm{M}]^{+}\right)$: 662.2933, found 663.2939. 


\section{Crossover experiment between 9 and 1-methylindole}

Platinum dimethyl complex 1a $(22.4 \mu \mathrm{mol}, 12.2 \mathrm{mg})$ was weighed out in an oven-dried screw-capped NMR tube in the drybox. TFE- $d_{3}(511 \mu \mathrm{L})$ and BF B $_{3}\left(0.477 \mathrm{M}\right.$ in TFE- $d_{3}, 24.6$ $\mu \mathrm{mol}, 51.6 \mu \mathrm{L}$ ) were then added. The suspension was removed from the drybox and sonicated until it became a homogeneous orange solution. The reaction was then thermally equilibrated to $30^{\circ} \mathrm{C}$ in a Varian mercury $300 \mathrm{MHz} \mathrm{NMR}$, and 2-methylindole $(0.183 \mathrm{M}$ in TFE- $d_{3}, 22.4 \mu \mathrm{mol}, 122 \mu \mathrm{L}$ ) was then injected. [9] was monitored, and reached a maximum in ca. 2 min. at which conversion of $\mathbf{2 a}$ to $\mathbf{9}$ was $\sim 90 \%$ complete. The tube was then removed from the instrument, 1-methylindole was injected, and the sample was re-inserted ( $30 \mathrm{sec}$.) An initial ratio of $81(1) \% \mathbf{9}, 10(1) \% \mathbf{1 0}, 9(1) \% \mathbf{1 1}$ was observed with 0(1)\% 2a. Kinetic data were recorded for 9.7 hours. After an additional 2 days at RT, 9 was completely converted to $\mathbf{1 0}\left(k_{\mathrm{RAR}}=9.1(3) \times 10^{-5} \mathrm{sec}^{-1}\right)$ and $\mathbf{1 1}$ was completely converted to $\mathbf{1 2}$. A ratio of 91(1)\% 10 to $9(1) \% 12$ was observed.

\section{Conversion of $6 \mathrm{a}$ to $8 \mathrm{a}$ at high $\left[\mathrm{H}^{+}\right]$}

Platinum dimethyl complex 1a $(22.7 \mu \mathrm{mol}, 12.4 \mathrm{mg})$ was weighed out in a $1 \mathrm{~mL}$ graduated cylinder in the drybox. $\mathrm{BF}_{3}\left(0.477 \mathrm{M}\right.$ in TFE- $d_{3}, 56.8 \mu \mathrm{mol}, 119 \mu \mathrm{L}$ ) and TFE- $d_{3}$ (to make $1.00 \mathrm{~mL}$ ) were then added. $480 \mu \mathrm{L}$ of this solution $\left(10.9 \mu \mathrm{mol} \mathrm{Pt}, 2.5\right.$ equiv. $\left.\mathrm{BF}_{3}\right)$ was then distributed into each of 2 screw-capped NMR tubes. Separately, indole (14.5 mg, $124 \mu \mathrm{mol})$, $\mathrm{BF}_{3}\left(0.477 \mathrm{M}\right.$ in TFE- $\left.d_{3}, 62.0 \mu \mathrm{mol}, 130 \mu \mathrm{L}\right)$. and TFE- $d_{3}(356 \mu \mathrm{L})$ were mixed in a $10 \mathrm{~mL}$ culture tube. $220 \mu \mathrm{L}$ of this solution (5 equiv. indole to $\mathrm{Pt}, 2.5$ equiv. $\mathrm{BF}_{3}$ to $\mathrm{Pt}$ ) was then distributed into each of two $1 \mathrm{~mL}$ syrenges. The NMR tubes and syrenges were removed from the drybox. One NMR tube was then thermally equilibrated to $40{ }^{\circ} \mathrm{C}$ in a Varian inova $500 \mathrm{MHz}$ NMR spectrometer. It was then ejected, and the content of 1 syrenge was added to the NMR tube (to make $10.9 \mu \mathrm{mol} \mathrm{Pt,} 5$ equiv. $\mathrm{BF}_{3}, 5$ equiv. indole, and $700 \mu \mathrm{L}$ TFE- $d_{3}$ ). The tube was shaken and re-inserted. After rapid re-shimming, kinetic data were recorded for 7.5 hours. A second trial was then conducted with the other NMR tube and syrenge. Reaction progress was monitored by NMR. Rate constants for this experiment are $k_{\text {binding }}=$ $5.35 \_10^{-4} \mathrm{~s}^{-1}$ and $k_{\mathrm{RAR}}=1.44 \_10^{-4} \mathrm{~s}^{-1}$, where $k_{\text {binding }}$ is the pseudo first order rate constant for the binding of indole to $\mathbf{2 a} .24$

\section{Comment regarding H/D Exchange and Methane Isotopologs Formed in the Conversion of 3 to 4}

In the conversion of $\mathbf{3 a}$ to $\mathbf{4 a}$ the methane that is formed is $65 \% \mathrm{CH}_{4}, 25 \% \mathrm{CH}_{3} \mathrm{D}$ due to $\mathrm{D}^{+}$ incorporation in the formation of $\mathbf{2 a}$ from 1a. A distribution of $53 \% \mathrm{CH}_{4}, 47 \% \mathrm{CH}_{3} \mathrm{D}$ is observed in the conversion of 3a- $d_{3}$ to $4 \mathbf{a}-d_{2}$. ${ }^{2} \mathrm{H}$ NMR spectra of $4 \mathbf{a}$ and $4 \mathbf{a}-d_{2}$ were recorded to track ${ }^{2} \mathrm{H}$ incorporation in these compounds (see Supporting Information). ${ }^{2} \mathrm{H}$ incorporation is observed in $\mathrm{C} 2-\mathrm{H}(6.8 \mathrm{ppm})$ in both $\mathbf{4 a}$ and, $\mathbf{4 a}-d_{2}$. Little ${ }^{2} \mathrm{H}$ incorporation is observed in $\mathrm{C} 1,3-\mathrm{H}$ (5.0 ppm) in 4a. As expected, ${ }^{2} \mathrm{H}$ is observed in this position in $\mathbf{4 a}-d_{2}$. A small amount of H/D exchange with solvent is observed in the diimine backbone (2.4 ppm), but none is observed in the mesityl methyl groups. Accordingly, the high level of $\mathrm{CH}_{4}$ relative to $\mathrm{CH}_{3} \mathrm{D}$ observed in the formation of $\mathbf{4 a}-d_{2}$ is attributed to some $\mathrm{H} / \mathrm{D}$ exchange with $\mathrm{C} 2-\mathrm{H}$ in the indenyl fragment prior to methane liberation and a low level of decomposition of the diimine ligand. 


\section{Supplementary Material}

Refer to Web version on PubMed Central for supplementary material.

\section{Acknowledgments}

This work was supported by the BP MC2 program and by the NIH (NRSA fellowship GM075691 to T.J.W.). These sponsors are gratefully acknowledged. We thank Dr. Tom G. Driver for helpful discussions, especially regarding the preparation and use of $\mathrm{BF}_{3}$-TFE- $d_{3}$.

\section{References and Notes}

1.

For recent reviews on C-H activation and functionalization, see: Arndtsen BA, Bergman RG, Mobley

TA, Peterson TH. Acc Chem Res. 1995; 28:154-162.Erker G. Angew Chem Int Ed. 1999; 38:1699_ 1712.Davies HML, Antoulinakis EG. J Organomet Chem. 2001; 617-618:47-55.Crabtree RH. J Chem Soc Dalton Trans. 2001; 17:2437-2450.Labinger JA, Bercaw JE. Nature. 2002; 417:507-514. [PubMed: 12037558] Fekl U, Goldberg KI. Adv Inorg Chem. 2003; 54:259-320.

2. Shilov AE, Shul'pin GB. Chem Rev. 1997; 97:2879-2932. [PubMed: 11851481]

3. Johansson L, Ryan OB, Tilset MJ. Am Chem Soc. 1999; 121:1974-1975.Johansson L, Tilset M. J Am Chem Soc. 2001; 123:739-740. [PubMed: 11456590]

4. (a) Johansson L, Tilset M, Labinger JA, Bercaw JE. J Am Chem Soc. 2000; 122:10846-10855.(b) Zhong AH, Labinger JA, Bercaw JE. J Am Chem Soc. 2002; 124:1378-1399. [PubMed: 11841307] (c) Heyduk AF, Driver TG, Labinger JA, Bercaw JE. J Am Chem Soc. 2004; 126:15034-15035. [PubMed: 15547993] (d) Driver TG, Day MW, Labinger JA, Bercaw JE. Organometallics. 2005; 24:3644-3654.(e) Owen JS, Labinger JA, Bercaw JE. J Am Chem Soc. 2006; 128:2005-2016. [PubMed: 16464102]

5. Driver, TG.; Labinger, JA.; Bercaw, JE. California Institute of Technology; Unpublished Results 6. Johansson L, Tilset M. J Am Chem Soc. 2001; 123:739-740. [PubMed: 11456590]

7. (a) Dunina VV, Zalevskaya OA, Potapov VM. Russian Chem Rev. 1988; 57:250-269.(b) Ryabov AD. Chem Rev. 1990; 90:403-424.

8.

Some examples include Dick AR, Hull KL, Sanford MS. J Am Chem Soc. 2004; 126:2300-2301.

[PubMed: 14982422] Desai LV, Hull KL, Sanford MS. J Am Chem Soc. 2004; 126:9542-9543.

[PubMed: 15291549] Tan KL, Bergman RG, Ellman JA. J Am Chem Soc. 2001; 123:2685-2686.

[PubMed: 11456947] Tan KL, Bergman RG, Ellman JA. J Am Chem Soc. 2002; 124:13964-13965.

[PubMed: 12440873] Dangel BD, Godula K, Youn SW, Sezen B, Sames D. J Am Chem Soc. 2002; 124:11856-11857. [PubMed: 12358522]

9.

Recent examples include Pd: Ferreira EM, Stoltz BM. J Am Chem Soc. 2003; 125:9578-9579.

[PubMed: 12904010] Abbiati G, Beccalli EM, Broggini G, Zoni C. J Org Chem. 2003; 68:76257628. [PubMed: 14510534] Pt: Liu C, Han X, Wang X, Widenhoefer RA. J Am Chem Soc. 2004; 126:3700-3701. [PubMed: 15038708]

10. Lane BS, Brown MA, Sames D. J Am Chem Soc. 2005; 127:8050-8057. [PubMed: 15926829]

11. Deprez NR, Kalyni D, Krause A, Sanford MS. J Am Chem Soc. 2006; 128:4972-4973. [PubMed: 16608329]

12. Kaminskaia NV, Ullmann GM, Fulton DB, Kostic' NM. Inorg Chem. 2000; 39:5004-5013. [PubMed: 11233196]

13. Osa T, Hino H, Fujieda S, Shiio T, Kono T. Chem Pharm Bull. 1986; 34:3563-3572. [PubMed: 3815589] 
14.

We believe that indole is bound through $\mathrm{N} 1$ as suggested by the authors, but is in the $3 H$-tautomer. ${ }^{1} \mathrm{H}$ NMR (300 MHz, dichloromethane- $\left.d_{2}\right) \delta: 8.37\left(\mathrm{~s}, J_{\mathrm{Pt}-\mathrm{H}}=30 \mathrm{~Hz}, 2 \mathrm{H}, \mathrm{C} 2-\mathrm{H}\right), 8.58\left(\mathrm{~d}, J_{\mathrm{Pt}-\mathrm{H}}=8.2\right.$ $\mathrm{Hz}, 2 \mathrm{H}), 7.62-7.08(\mathrm{~m}, 6 \mathrm{H}), 4.03\left(\mathrm{~s}, 4 \mathrm{H}, \mathrm{C} 3-\mathrm{H}_{2}\right)$.

15.

For example: indole $+\mathrm{Pt}^{0}\left(\mathrm{PEt}_{3}\right)_{4} \rightarrow$ trans- $\left[\left(\mathrm{PEt}_{3}\right)_{2} \mathrm{Pt}^{\mathrm{II}}(\mathrm{H})(1\right.$-indolyl $\left.)\right]+2$ PEt 3 . Chantson JT, Lotz S. J

Organomet Chem. 2004; 689:1315-1324.

16.

$\left[\left(\eta^{6}-\mathrm{C}_{8} \mathrm{H}_{7} \mathrm{~N}\right) \mathrm{ML}_{n}\right]$ complexes are known for several metals. Common examples include $\mathrm{ML}_{\mathrm{n}}=$ $\mathrm{Cr}(\mathrm{CO})_{3}$, see Fischer EO, Goodwin HA, Kreiter CG, Simmons HD, Sonogashira K, Wild SB. J Organomet Chem. 1968; 14:359-374.Nesmeyanov AN, Ustynyuk NA, Thoma T, Prostakov NS, Soldatenkov AT, Pleshakov VG, Urga K, Ustynyuk YA, Trifonova OI, Oprunenko YF. J Organomet Chem. 1982; 231:5-24.ML $=\mathrm{CpRu}^{+}$, see Moriarty RM, Ku YY, Gill US. Organometallics. 1988; 7:660-665.Other examples include $\mathrm{M}=\mathrm{Mn}^{\mathrm{I}}, \mathrm{Co}^{\mathrm{III}}, \mathrm{Rh}^{\mathrm{I}}, \mathrm{Rh}^{\mathrm{III}}$, $\mathrm{Ir}^{\mathrm{I}}$, and $\mathrm{Ir}^{\mathrm{III}}$. [ $\left.\left(\eta^{5}-\mathrm{C}_{8} \mathrm{H}_{6} \mathrm{~N}\right) \mathrm{ML}_{n}\right]$ complexes are known for $\mathrm{ML}_{\mathrm{n}}=\mathrm{Mn}(\mathrm{CO})_{3}{ }^{+}$, see Ji LN, Kershner DL, Rerek ME, Basolo F. J Organomet Chem. 1985; 296:83-94.for $\mathrm{ML}_{\mathrm{n}}=\mathrm{Cp} \mathrm{Ir}^{2+}$, see White C, Thompson SJ, Maitlis PMJ. Chem Soc Dalton Trans. 1977:1654-1661.Other examples include M $=\mathrm{Ti}^{\mathrm{IV}}, \mathrm{Mo}^{\mathrm{II}}$, and $\mathrm{Fe}^{\mathrm{II}}$.

17.

Analyzed as in Romeo R, Monsu Scolaro L, Nastasi N, Arena G. Inorg Chem. 1996; 35:5087-5096.

[PubMed: 11666719]

18.

Error determined by established methods. See Morse PM, Spencer MD, Wilson SR, Girolami GS.

Organometallics. 1994; 13:1646-1655.

19. Yasuda M, Pak C, Sakurai H. Bull Chem Soc Japan. 1980; 53:502-507.

20.

Some [( $3 \mathrm{H}$-indole)MLn] complexes are known for other metals. For examples of [Re] complexes, see Johnson TJ, Arif AM, Gladysz JA. Organometallics. 1994; 13:3182-3193.; for [Ir] see Chen S, Noll BC, Peslherbe L, Rakowski DuBois M. Organometallics. 1997; 16:1089-1092.

21.

C3-H in 6a can be observed at early stages of kinetic runs $\left(\delta=5.95 \mathrm{ppm}, J_{\mathrm{Pt}-\mathrm{H}}=87 \mathrm{~Hz}(500 \mathrm{MHz})\right)$, but N1-H was never found for coordinated indole.

22.

A reviewer proposed an alternative $\mathrm{C} 3 \sigma$-bound structure for $6 \mathrm{a}, 9$, and 11 , but we favor the $\pi$ complexes as shown. The $\sigma$-bound structure is inconsistent with ${ }^{1} \mathrm{H}$ and ${ }^{13} \mathrm{C}$ chemical shifts for $\mathrm{C} 3-\mathrm{H}$ : $\delta \mathrm{C} 3-\mathrm{H}=5.4$ and 6.0 for compounds $6 \mathrm{a}$ and 11 , respectively, as identified by $J_{\mathrm{Pt}-\mathrm{H}}$ and $\mathrm{H} / \mathrm{D}$ exchange. This is more consistent with an $\mathrm{sp}^{2}$ carbon center. Moreover, no ${ }^{13} \mathrm{C}$ signal is present in the 175-180 region of the spectrum of 11 , as would be needed for $\mathrm{C} 2$ in the $\sigma$-bound structure. It is likely, however, that the platinum center is more tightly associated with the more electron rich $\mathrm{C} 3$ side of the olefin. This is a possible explanation for the low $J_{\mathrm{Pt}-\mathrm{H}}$ for $\mathrm{C} 2$ in 11.

Organometallics. Author manuscript; available in PMC 2016 April 15. 


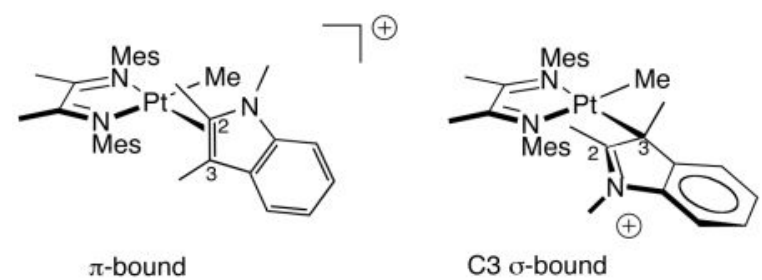

23.

Reactions of $\mathrm{N}$-protected indoles (Ac, Tf, Ts), and benzofuran afforded complex mixtures of $\mathrm{C}-\mathrm{H}$ activation products. Pyrrole, carbazole, and 3-methylindole afforded complex mixtures of coordination complexes of the general form $\left[(\text { diimine }) \mathrm{Pt}(\mathrm{Me})\left(\mathrm{C}_{\mathrm{n}} \mathrm{H}_{\mathrm{n}} \mathrm{N}-d_{\mathrm{n}}\right)\right]^{+}$. Hydrogenated homologs of indene and indole also react with $2 \mathrm{a}$. Indane forms a mixture of $\mathrm{C}-\mathrm{H}$ activation products, which includes an $\eta^{3}$-benzyl complex analogous to that obtained from ethylbenzene; see notes $4 \mathrm{~cd}$. 1,2-Dihydroindole forms a stable $N$-bound coordination complex, which has ${ }^{1} \mathrm{H}$ NMR (300 MHz, TFE- $\left.d_{3}\right) \delta:$ 7.21-7.14 (m, 3H), 7.07-7.06 (m, 4H), $6.89(\mathrm{~s}, 1 \mathrm{H}), 3.47-3.30(\mathrm{~m}, 2 \mathrm{H})$, 2.87-2.75 (m, 1H), 2.58-2.47 (m, 1H), $2.33(\mathrm{~s}, 3 \mathrm{H}), 2.30(\mathrm{~s}, 6 \mathrm{H}), 2.17(\mathrm{~s}, 3 \mathrm{H}), 2.17(\mathrm{~s}, 3 \mathrm{H}), 1.93(\mathrm{~s}$, $3 \mathrm{H}), 1.80(\mathrm{~s}, 3 \mathrm{H}), 1.75(\mathrm{~s}, 3 \mathrm{H}), 0.53\left(\mathrm{~s}, J_{\mathrm{Pt}-\mathrm{H}}=76 \mathrm{~Hz}, 3 \mathrm{H}\right)$.

24.

Rate constants for this experiment were determined by modeling [6a] as an intermediate in a scheme of consecutive reactions. For the reaction scheme $2 \mathrm{a}+$ indole (excess) $-k_{\text {binding }} \rightarrow 6 \mathrm{a}-k_{\mathrm{RAR}} \rightarrow$ products, $[6 \mathrm{a}] /[6 \mathrm{a}]_{\mathrm{o}}=\left(k_{\text {binding }} /\left(k_{\text {binding }}+k_{\mathrm{RAR}}\right)\right)\left(\mathrm{e}^{-k_{\text {binding }} \mathrm{t}}-\mathrm{e}^{-k_{\mathrm{RAR}}{ }^{\mathrm{t}}}\right)$ See Laidler, K. J. Chemical Kinetics, $3^{\text {rd }}$ ed:; Harper \& Row: New York, 1987.

25. Brown HC, Johannesen RB. J Am Chem Soc. 1953; 75:16-20.

26.

Observed $J_{\mathrm{Pt}-\mathrm{H}}=22 \mathrm{~Hz}$ for this signal in a spectrum acquired at $300 \mathrm{MHz}$.

27.

This signal (C3-H) is rapidly deuterated under the reaction conditions, but can be observed at the beginning of a kinetics run. 

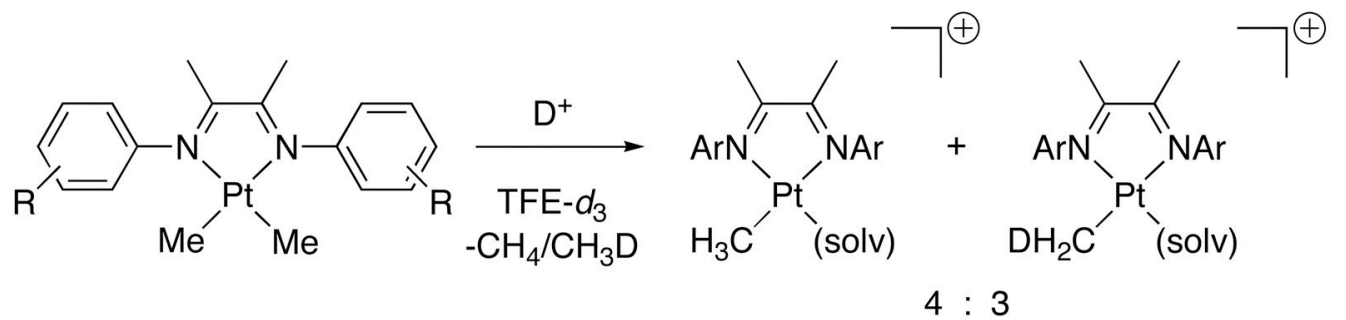

1a: $\mathrm{R}=2,4,6-\mathrm{Me}_{3}-$

$2 a$

1b: $\mathrm{R}=3,5-\mathrm{Me}_{2}-$

$2 b$

1c: $\mathrm{R}=3,5^{-}\left(\mathrm{CF}_{3}\right)_{2}-$

2c

1d: $\mathrm{R}=3,5-\mathrm{tBu}_{2}$

2d

1e: $\mathrm{R}=2,6-\mathrm{Me}_{2}$

$2 e$
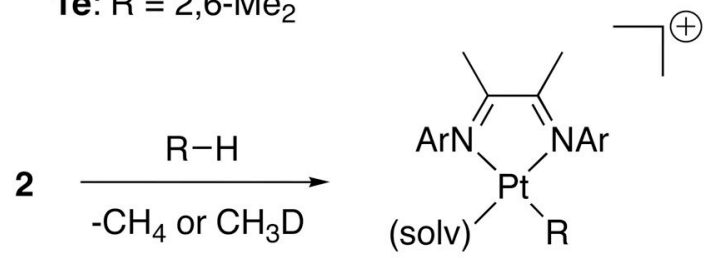

Scheme 1. 


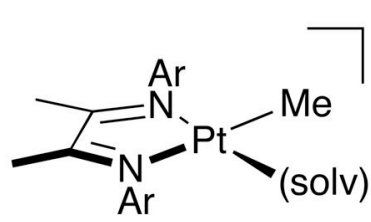

$2 a, 2 b, 2 c, 2 d$
(†)

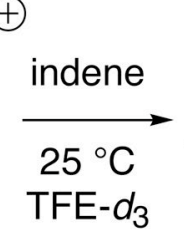

3a, 3b, 3c, 3d

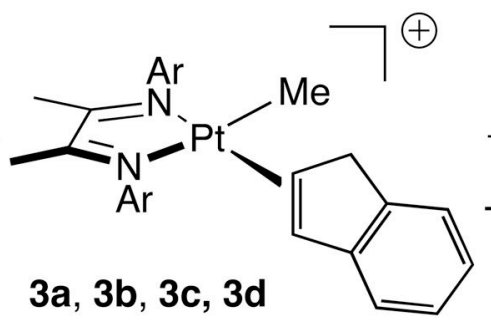

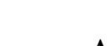
- methane

Scheme 2. 

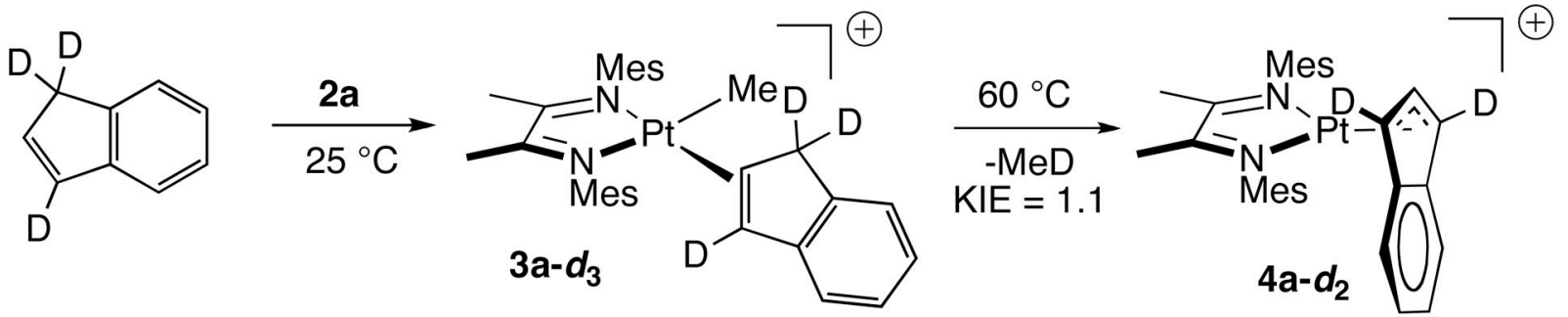

Scheme 3. 

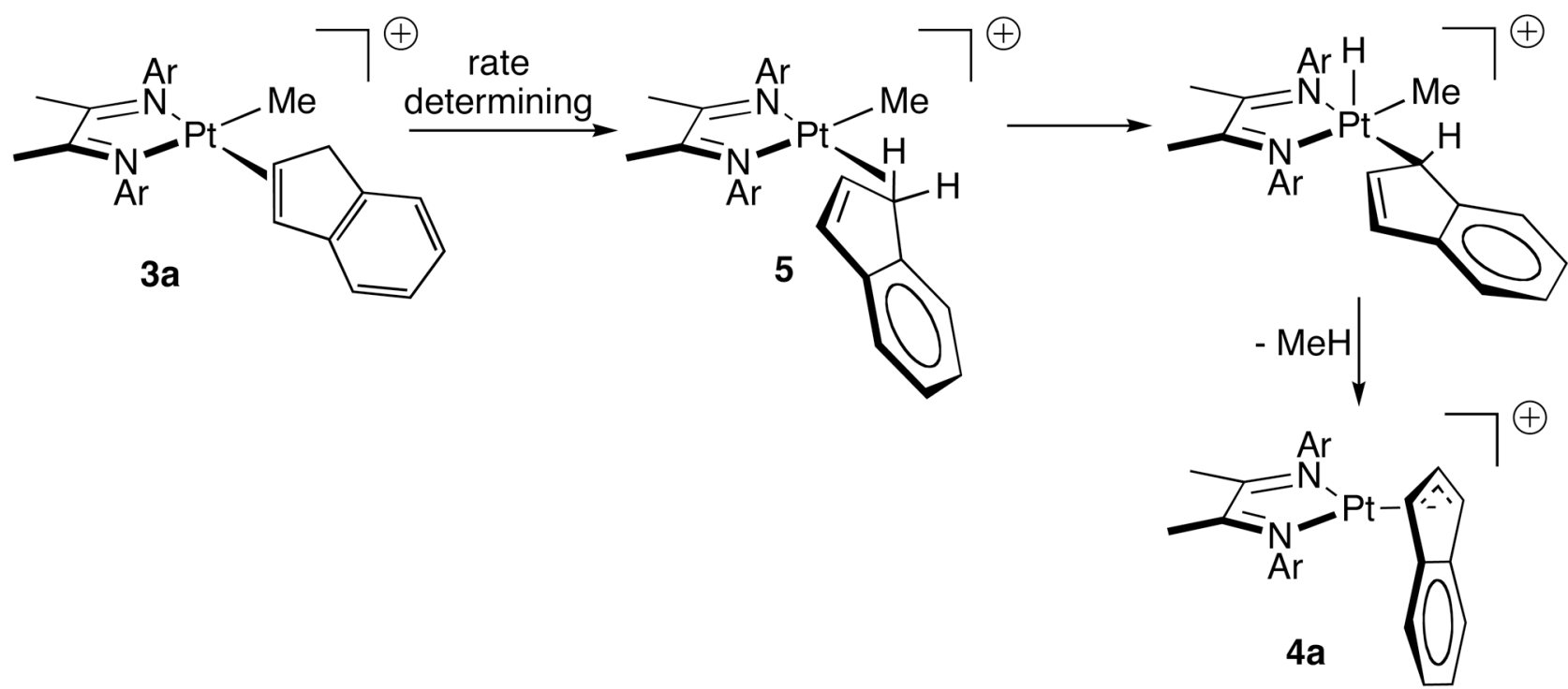

Scheme 4. 

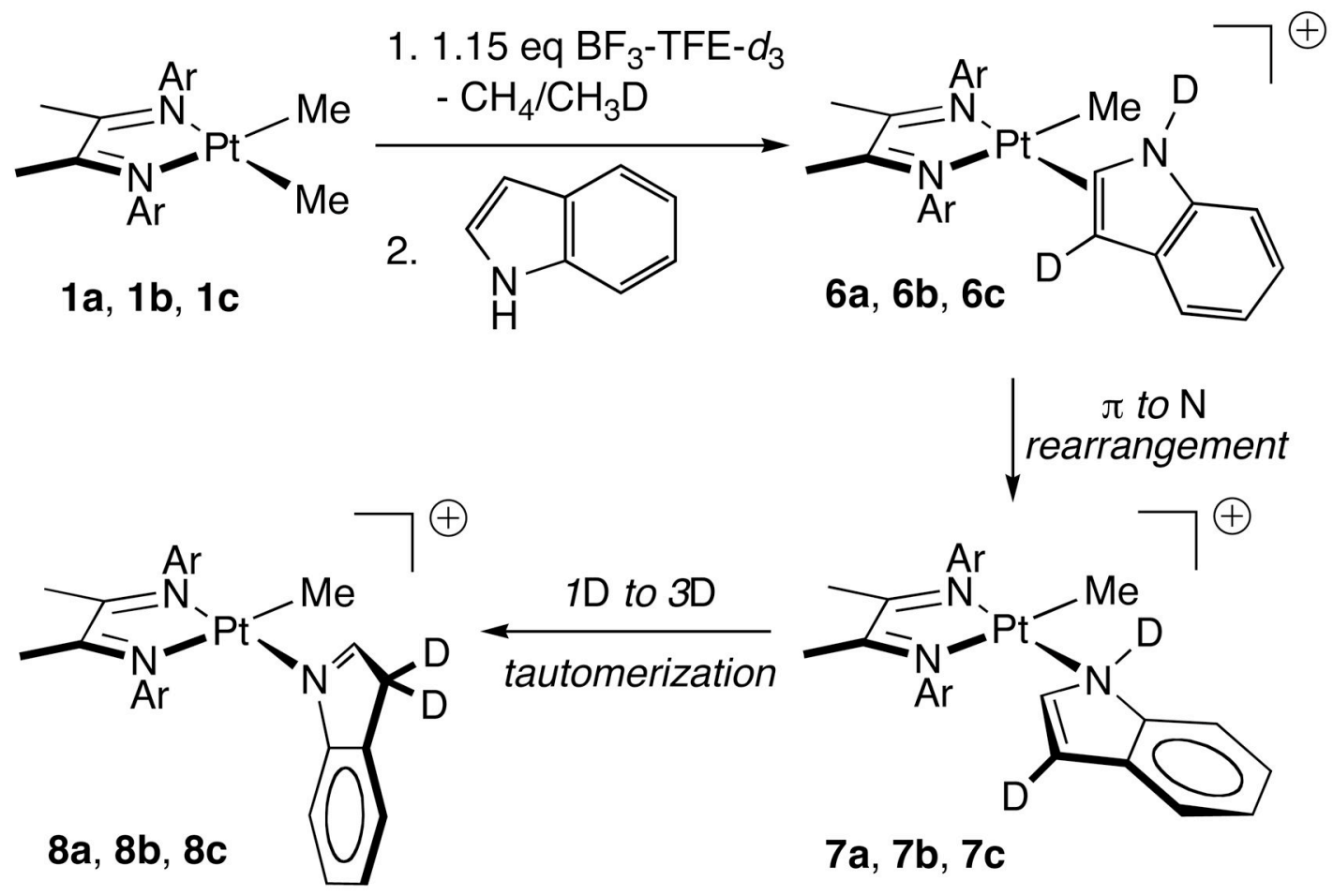

$7 a, 7 b, 7 c$

Scheme 5. 

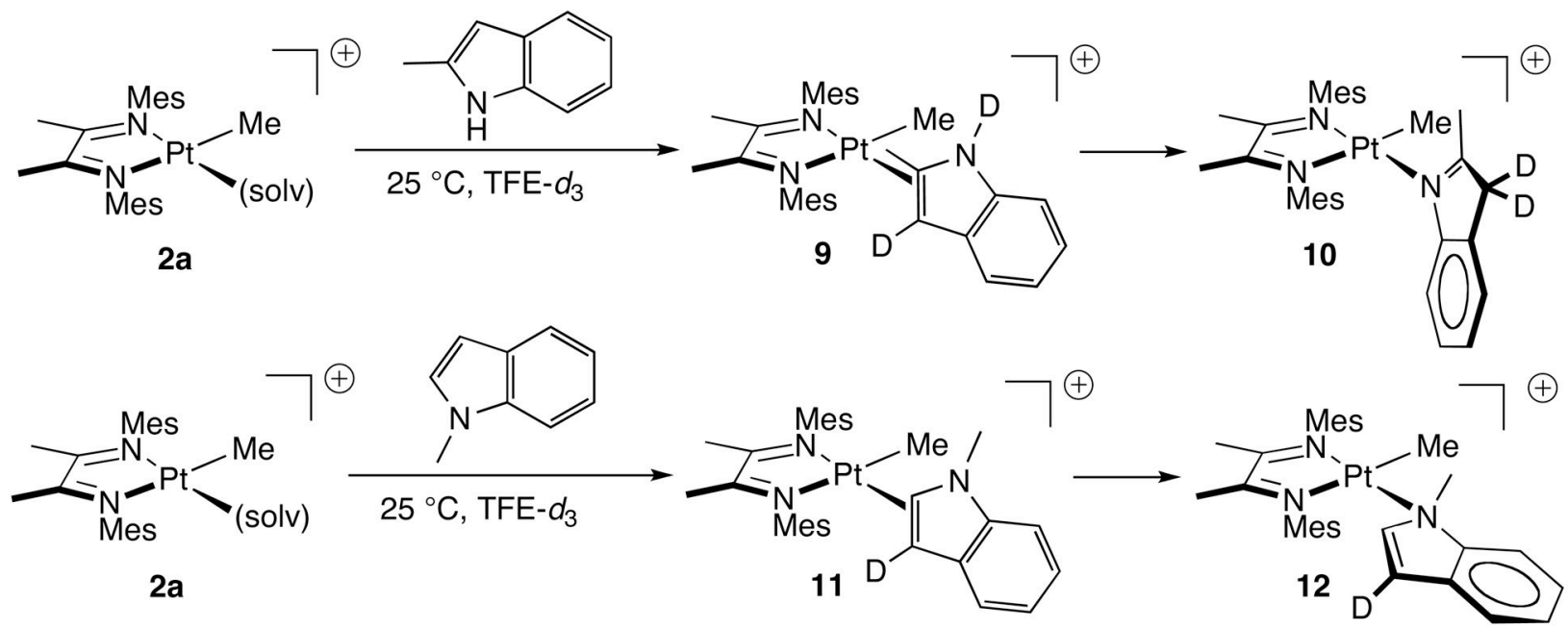

Scheme 6. 


\section{Table 1}

First-order rate constants for the conversion of indene $\pi$ complex 3a to $\eta 3$-indenyl complex $4 \mathbf{a}$.

\begin{tabular}{llll}
\hline Entry & $\mathbf{T}\left({ }^{\circ} \mathbf{C}\right)$ & $\boldsymbol{k}\left(\mathbf{s}^{-\mathbf{1}}\right)$ & $\mathbf{t}_{(\mathbf{1} / \mathbf{2})}(\mathbf{m i n})$ \\
\hline 1 & 50 & $3.38(11) \times 10^{-5}$ & $342(11)$ \\
2 & 60 & $1.31(9) \times 10^{-4}$ & $88.5(58)$ \\
3 & 70 & $4.60(18) \times 10^{-4}$ & $25.1(10)$ \\
4 & 80 & $1.84(10) \times 10^{-3}$ & $6.28(32)$ \\
5 & 90 & $5.10(10) \times 10^{-3}$ & $2.28(38)$ \\
\hline
\end{tabular}




\section{Table 2}

NMR data for Pt-Me groups of TFE adduct 2a, indene $\pi$ complex 3a, and the $\pi$ and $N$-indole adducts.

\begin{tabular}{lccl}
\hline Complex & ${ }^{\mathbf{1}} \mathbf{H} \delta^{\boldsymbol{a}}(\mathbf{p p m})$ & ${ }^{\mathbf{1 3}} \mathbf{C} \delta(\mathbf{p p m})$ & $\boldsymbol{J}_{\mathbf{P t}-\mathbf{H}} \boldsymbol{b}_{(\mathbf{H z})}$ \\
\hline $\mathbf{2 a}(\mathrm{TFE})^{c}$ & +0.66 & 21.5 & 72 \\
$\mathbf{3 a}$ (indene) & -0.74 & 4.7 & 72 \\
$\mathbf{6 a}$ (indole $\pi)$ & -1.02 & $-d$ & $62^{a}$ \\
$\mathbf{8 a}($ indole $N)$ & 0.74 & -12.1 & 78 \\
$\mathbf{9}$ & -0.75 & -3.6 & 73 \\
$\mathbf{1 0}$ & 0.53 & -13.7 & 78 \\
$\mathbf{1 1}$ & -1.16 & -3.0 & 73 \\
$\mathbf{1 2}$ & 0.26 & -16.8 & 78 \\
\hline
\end{tabular}

${ }^{a}$ Data recorded at 500 or $600 \mathrm{MHz}$.

$b_{\text {Recorded at }} 300 \mathrm{MHz}$.

$c_{\text {Notes }} 4 \mathrm{bc}$.

$d_{\text {The lifetime of this species is too short to record }}{ }^{13} \mathrm{C}$ NMR. 


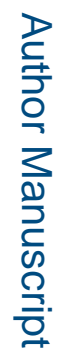
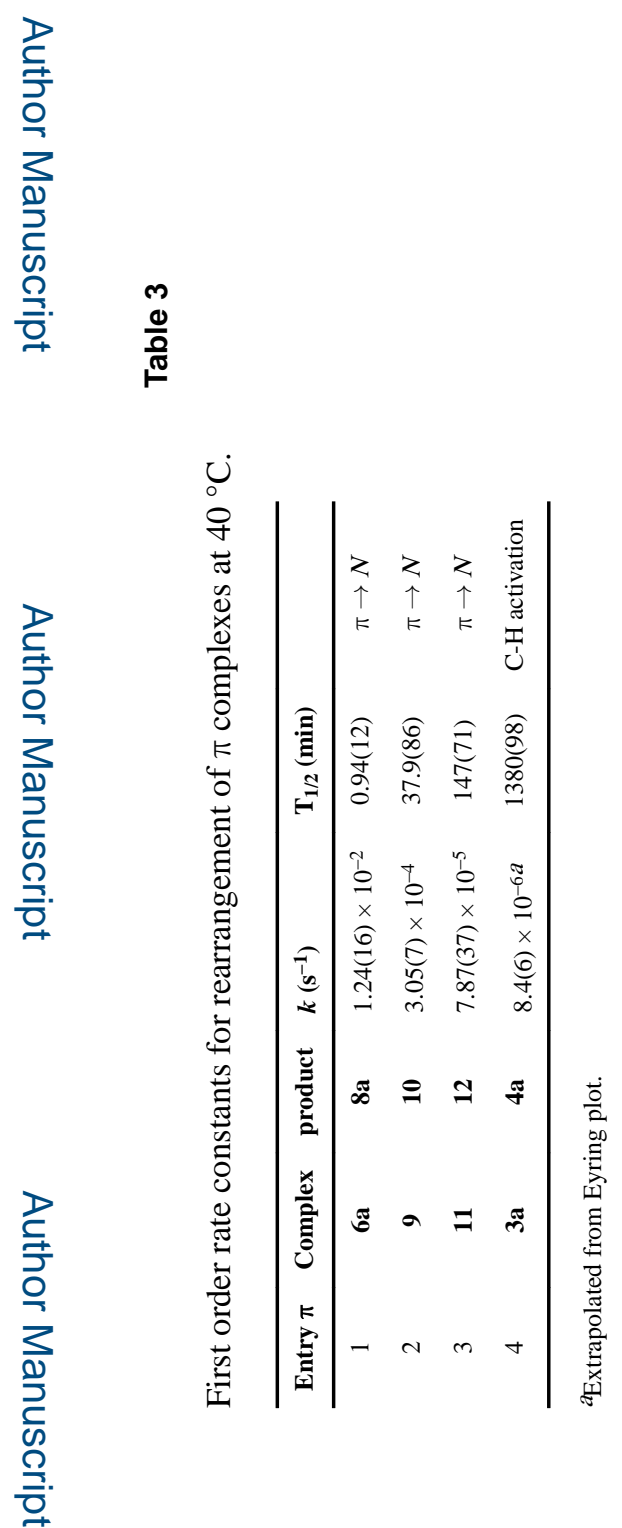

Organometallics. Author manuscript; available in PMC 2016 April 15. 


\section{Table 4}

Acid dependence of rate constant for rearrangement $\mathbf{6 a}$.

\begin{tabular}{llll}
\hline Entry & $\mathbf{B F}_{\mathbf{3}}$ & $\boldsymbol{k}\left(\mathbf{s}^{\mathbf{- 1}}\right)$ & $\mathbf{t}_{\mathbf{1} / \mathbf{2}}(\mathbf{m i n})$ \\
\hline 1 & 1.1 equiv. & $1.24(16) \times 10^{-2}$ & $0.94(12)$ \\
2 & 2.5 equiv. & $1.09(21) \times 10^{-2}$ & $1.09(19)$ \\
3 & 4.0 equiv. & $3.51(12) \times 10^{-3}$ & $3.29(11)$ \\
$4^{a}$ & 5.0 equiv. & $1.48(5) \times 10^{-4}$ & $78.3(27)$ \\
\hline
\end{tabular}

Conditions: $\mathrm{BF}_{3}$ is added to 1a in TFE. 5 equivalents of indole in TFE is then injected.

${ }^{a}$ Indole binding and tautomerization are slow relative to $k \mathrm{RAR}$. 